TRANSACTIONS OF THE

AMERICAN MATHEMATICAL SOCIETY

Volume 354, Number 8, Pages 3229-3262

S 0002-9947(02)02976-8

Article electronically published on April 3, 2002

\title{
TRANSFER FUNCTIONS \\ OF REGULAR LINEAR SYSTEMS \\ PART II: THE SYSTEM OPERATOR AND THE LAX-PHILLIPS SEMIGROUP
}

\author{
OLOF STAFFANS AND GEORGE WEISS
}

\begin{abstract}
This paper is a sequel to a paper by the second author on regular linear systems (1994), referred to here as "Part I". We introduce the system operator of a well-posed linear system, which for a finite-dimensional system described by $\dot{x}=A x+B u, y=C x+D u$ would be the $s$-dependent matrix $S_{\Sigma}(s)=\left[\begin{array}{cc}A-s I & B \\ C & D\end{array}\right]$. In the general case, $S_{\Sigma}(s)$ is an unbounded operator, and we show that it can be split into four blocks, as in the finite-dimensional case, but the splitting is not unique (the upper row consists of the uniquely determined blocks $A-s I$ and $B$, as in the finite-dimensional case, but the lower row is more problematic). For weakly regular systems (which are introduced and studied here), there exists a special splitting of $S_{\Sigma}(s)$ where the right lower block is the feedthrough operator of the system. Using $S_{\Sigma}(0)$, we give representation theorems which generalize those from Part I to well-posed linear systems and also to the situation when the "initial time" is $-\infty$. We also introduce the Lax-Phillips semigroup $\mathfrak{T}$ induced by a well-posed linear system, which is in fact an alternative representation of a system, used in scattering theory. Our concept of a Lax-Phillips semigroup differs in several respects from the classical one, for example, by allowing an index $\omega \in \mathbb{R}$ which determines an exponential weight in the input and output spaces. This index allows us to characterize the spectrum of $A$ and also the points where $S_{\Sigma}(s)$ is not invertible, in terms of the spectrum of the generator of $\mathfrak{T}$ (for various values of $\omega$ ). The system $\Sigma$ is dissipative if and only if $\mathfrak{T}$ (with index zero) is a contraction semigroup.
\end{abstract}

\section{INTRODUCTION}

This is a continuation of the paper Weiss [41], which addressed some fundamental questions about well-posed linear systems and, in particular, regular linear systems. A well-posed linear system is a linear system whose input, state and output spaces are Hilbert spaces, whose input and output functions are locally $L^{2}$, and for which on any finite time-interval, the final state and the output function depend continuously on the initial state and the input function. Certain functional equations must be satisfied, which express time-invariance and causality. If the transfer function of a well-posed system has a strong limit at $+\infty$, then the system is called regular.

Received by the editors February 23, 2001 and, in revised form, November 16, 2001.

2000 Mathematics Subject Classification. Primary 93C25; Secondary 34L25, 37L99, 47D06.

Key words and phrases. Well-posed linear system, (weakly) regular linear system, operator semigroup, system operator, generating operators, well-posed transfer function, scattering theory, Lax-Phillips semigroup, dissipative system. 
The precise definitions of these and other concepts used in the Introduction were given in [41], and we shall recall them in Sections 2 and 4 .

The concept of a well-posed linear system was introduced by Salamon [28], and equivalent definitions have since been used by Arov and Nudelman [1], Staffans 30, 31, and Weiss [40, 41. Regular linear systems were introduced in [40], and most well-posed systems encountered in applications are regular. There is now a rich literature using this concept, ranging from partial differential equations to engineering applications, see for instance Avalos et al. [2, Hinrichsen and Pritchard [8], Jacob and Zwart [10, Logemann et al. [14], Logemann and Ryan [15], Logemann, Ryan and Townley [16, 17], Logemann and Townley [18 19], Morris [20, Rebarber 25, 26], Rebarber and Townley 27, Staffans [30, 32, 33, 34], Weiss and Curtain [44, Weiss and Häfele [46] and Weiss ${ }^{2}$ 45].

The concept of a well-posed linear system is closely related to that of a scattering semigroup, as studied in Lax and Phillips [12, 13] - we shall say more about this connection later. Related definitions have been used by Helton [6], Ober and Montgomery-Smith [21], Ober and Wu [22, Yamamoto [47, and others.

To make our aims more easily understood, we explain what some of our results mean for a finite-dimensional linear system $\Sigma$ described by

$$
\begin{aligned}
& \dot{x}(t)=A x(t)+B u(t), \\
& y(t)=C x(t)+D u(t) .
\end{aligned}
$$

Here $u(\cdot)$ is the input function, $x(t)$ is the state at time $t$, and $y(\cdot)$ is the output function. We call the matrices $A, B, C, D$ the generating operators of $\Sigma$, and

$$
S_{\Sigma}(s)=\left[\begin{array}{cc}
A-s I & B \\
C & D
\end{array}\right] \quad(\text { where } s \in \mathbb{C})
$$

is called the system operator of $\Sigma$. The transfer function of this system is

$$
\mathbf{G}(s)=C(s I-A)^{-1} B+D \quad(\text { for } s \in \rho(A)) .
$$

Note that for $s \in \rho(A)$ we have the Schur factorization

$$
S_{\Sigma}(s)=\left[\begin{array}{cc}
A-s I & 0 \\
C & I
\end{array}\right]\left[\begin{array}{cc}
(A-s I)^{-1} & 0 \\
0 & \mathbf{G}(s)
\end{array}\right]\left[\begin{array}{cc}
A-s I & B \\
0 & I
\end{array}\right],
$$

which shows that (for $s \in \rho(A)$ ) $\operatorname{dim} \operatorname{Ker} \mathbf{G}(s)=\operatorname{dim} \operatorname{Ker} S_{\Sigma}(s)$. A point $z \in \mathbb{C}$ where $\operatorname{dim} \operatorname{Ker} S_{\Sigma}(z)$ is larger than its minimal value over all $s \in \mathbb{C}$ is called an invariant zero of $\Sigma$, see for example Zhou et al. [48. There are only finitely many such points $z$ in $\mathbb{C}$. In the particular situation when there exists an $s \in \mathbb{C}$ such that $S_{\Sigma}(s)$ is invertible (this implies an equal number of input and output components), the invariant zeros of $\Sigma$ are the points $z$ where $S_{\Sigma}(z)$ is not invertible.

Take $\tau \geq 0$. We are usually interested in the solutions of (1.1) for $t \in[0, \tau]$, but of course, the solutions exist on the whole real line. Given an initial state $x(0)$ and the restriction of $u$ to $[0, \tau]$, denoted by $\mathbf{P}_{\tau} u$, we can solve (1.1) to compute $x(\tau)$ and the restriction of $y$ to $[0, \tau]$, denoted by $\mathbf{P}_{\tau} y$. Formally, we have

$$
\left[\begin{array}{l}
x(\tau) \\
\mathbf{P}_{\tau} y
\end{array}\right]=\left[\begin{array}{ll}
\mathbb{T}_{\tau} & \Phi_{\tau} \\
\Psi_{\tau} & \mathbb{F}_{\tau}
\end{array}\right]\left[\begin{array}{l}
x(0) \\
\mathbf{P}_{\tau} u
\end{array}\right] .
$$


The operators appearing in the block $2 \times 2$ matrix above are given by

$$
\begin{aligned}
\mathbb{T}_{\tau} & =e^{A \tau}, & \Phi_{\tau} u & =\int_{0}^{\tau} e^{A(\tau-\sigma)} B u(\sigma) \mathrm{d} \sigma, \\
\left(\Psi_{\tau} x_{0}\right)(t) & =C e^{A t} x_{0}, & \left(\mathbb{F}_{\tau} u\right)(t) & =C \int_{0}^{t} e^{A(t-\sigma)} B u(\sigma) \mathrm{d} \sigma+D u(t),
\end{aligned}
$$

where $t \in[0, \tau]$. These families of operators (parametrized by $\tau \geq 0$ ) constitute an alternative description of the system $\Sigma$. This is, of course, a much more cumbersome description of $\Sigma$ than (1.1), but for infinite-dimensional systems these operator families are the natural starting point, see [41] or [31].

Let $U, X$ and $Y$ be the input, state and output space of the system $\Sigma$ from (1.1) (so that now these spaces are finite-dimensional) and denote $\mathcal{U}=L^{2}([0, \infty) ; U)$, $\mathcal{Y}=L^{2}((-\infty, 0] ; Y)$. For any $t \geq 0$, we denote by $\mathcal{S}_{-t}$ the left shift by $t$ on $\mathcal{Y}$ (this is the restriction of the bilateral left shift to $\mathcal{Y}$ ) and we denote by $\mathbf{S}_{t}^{*}$ the left shift by $t$ on $\mathcal{U}$. We define on $\mathcal{Y} \times X \times \mathcal{U}$ the operator $\mathfrak{T}_{t}$ by

$$
\boldsymbol{T}_{t}=\left[\begin{array}{ccc}
\mathcal{S}_{-t} & 0 & 0 \\
0 & I & 0 \\
0 & 0 & \mathbf{S}_{t}^{*}
\end{array}\right]\left[\begin{array}{ccc}
I & \Psi_{t} & \mathbb{F}_{t} \\
0 & \mathbb{T}_{t} & \Phi_{t} \\
0 & 0 & I
\end{array}\right]
$$

Then $\mathfrak{T}=\left(\mathfrak{T}_{t}\right)_{t \geq 0}$ is a strongly continuous semigroup on $\mathcal{Y} \times X \times \mathcal{U}$, called the Lax-Phillips semigroup (of index zero) induced by $\Sigma$. We see that this semigroup contains every operator from (1.5), so that it contains all the information about $\Sigma$. The intuitive interpretation of the space $\mathcal{Y} \times X \times \mathcal{U}$ and of $\mathfrak{T}_{t}$ acting on it is that the first component is the past output, the second component is the current state, while the third component is the future input. Indeed, let $y_{0} \in \mathcal{Y}, x_{0} \in X, u_{0} \in \mathcal{U}$, and for $t \geq 0$ define

$$
\left[\begin{array}{l}
y_{t} \\
x_{t} \\
u_{t}
\end{array}\right]=\boldsymbol{T}_{t}\left[\begin{array}{l}
y_{0} \\
x_{0} \\
u_{0}
\end{array}\right]
$$

Let $x$ and $y$ be the state trajectory and the output function of $\Sigma$, i.e., the solutions of (1.1) corresponding to the initial state $x(0)=x_{0}$ and the input function $u$. We extend $y$ to $\mathbb{R}$ by putting $y(t)=y_{0}(t)$ for $t \leq 0$. Then

$$
y(t-\xi)=y_{t}(-\xi), \quad x(t)=x_{t}, \quad u(t+\xi)=u_{t}(\xi)
$$

for all $t \geq 0$ and for almost every $\xi \geq 0$.

We have for almost every $t \geq 0$ (using the above notation)

$$
\frac{\mathrm{d}}{\mathrm{d} t}\left\|\left[\begin{array}{c}
y_{t} \\
x_{t} \\
u_{t}
\end{array}\right]\right\|^{2}=\|y(t)\|^{2}+\frac{\mathrm{d}}{\mathrm{d} t}\|x(t)\|^{2}-\|u(t)\|^{2} .
$$

The system $\Sigma$ is called dissipative if

$$
\frac{\mathrm{d}}{\mathrm{d} t}\|x(t)\|^{2} \leq\|u(t)\|^{2}-\|y(t)\|^{2} .
$$

Thus, $\Sigma$ is dissipative if and only if $\boldsymbol{T}$ is a contraction semigroup. In terms of the matrices $A, B, C, D$, the system $\Sigma$ is dissipative if and only if

$$
\left[\begin{array}{cc}
A+A^{*}+C^{*} C & B+C^{*} D \\
B^{*}+D^{*} C & D^{*} D-I
\end{array}\right] \leq 0 .
$$


Proposition 1.1. With the notation from (1.2) and (1.7), let $\mathfrak{A}$ be the generator of $\mathfrak{T}$. The spectrum $\sigma(\mathfrak{A})$ contains the imaginary axis. A point in the open right half-plane is in $\sigma(\mathfrak{A})$ if and only if it is in $\sigma(A)$, while a point $z$ in the open left half-plane is in $\sigma(\mathfrak{A})$ if and only if $S_{\Sigma}(z)$ is not invertible.

Hence, if $U$ and $Y$ have unequal dimensions, then every point in the left halfplane is in $\sigma(\mathfrak{A})$. On the other hand, if $S_{\Sigma}(s)$ is invertible for some $s \in \mathbb{C}$, then a point in the left half-plane is in $\sigma(\mathfrak{A})$ if and only if it is an invariant zero of $\Sigma$. Everything written starting with (1.7) and up to (1.8) (exclusive) remains valid for well-posed linear systems. The characterization (1.8) of dissipativity remains valid only under restrictive assumptions (see Remark 7.6) and in general the characterization is more complicated (see Theorem 7.4). Proposition 1.1 remains valid practically unchanged for well-posed systems, and this is a consequence of Theorem 6.3 .

Some of our results cannot be illustrated in the finite-dimensional situation, because they become trivialities. They concern the representation of well-posed systems by formulas similar to (1.1)-(1.3) (Theorems 3.1 and 3.2). A major hurdle is that, in general, the operators involved are unbounded and there is no natural decomposition of the second row of $S_{\Sigma}(s)$, which is denoted $C \& D$, into an operator with two components, such as $[C D]$. We show that such decompositions always exist, but they are not unique in general (see Corollary 3.5). In Section 4 we introduce and study weakly regular systems, for which there is a privileged decomposition of $C \& D$ and for which the representation formulas become simpler. Section [5]concerns the behavior of well-posed systems on the time interval $(-\infty, \infty)$ (i.e., with initial time $-\infty$ ). Section 6 is devoted to the Lax-Phillips semigroup and its generator, and in Section 7 we prove some basic facts about dissipative systems.

We will often use the terminology and results from [41, which we refer to as "Part I". In such cases, we put the prefix I in front of the number of the item quoted. For example, Definition I.2.1 refers to Definition 2.1 in Part I, and (I.4.8) refers to formula (4.8) in Part I.

\section{WELL-POSED LINEAR SYSTEMS}

In this section we review some concepts and results about well-posed linear systems from Part I. We do not give proofs.

Notation 2.1. Let $W$ be a Hilbert space. We regard $L_{\text {loc }}^{2}((-\infty, \infty) ; W)$ as a Fréchet space, with the metric generated by the seminorms

$$
\left\|u_{n}\right\|=\left(\int_{-n}^{n}\|u(t)\|^{2} \mathrm{~d} t\right)^{1 / 2}, \quad n \in \mathbb{N}
$$

For any interval $J$, we regard $L_{\text {loc }}^{2}(J ; W)$ as a subspace of $L_{\text {loc }}^{2}((-\infty, \infty) ; W)$ (identifying $L_{\text {loc }}^{2}(J ; W)$ with the set of functions in $L_{\text {loc }}^{2}((-\infty, \infty) ; W)$ which vanish outside of $J)$, and similarly we regard $L^{2}(J ; W)$ as a subspace of $L^{2}((-\infty, \infty) ; W)$. Of course, if $J$ is bounded, then $L_{\text {loc }}^{2}(J ; W)=L^{2}(J ; W)$. Let $\mathbf{P}_{J}$ be the projection of $L_{\mathrm{loc}}^{2}((-\infty, \infty) ; W)$ onto $L_{\mathrm{loc}}^{2}(J ; W)$ (by truncation). We abbreviate $\mathbf{P}_{\tau}=\mathbf{P}_{[0, \tau]}$ (where $\tau \geq 0), \mathbf{P}_{-}=\mathbf{P}_{(-\infty, 0]}$ and $\mathbf{P}_{+}=\mathbf{P}_{[0, \infty)}$. The operator $\mathbf{S}_{\tau}$ is the (unilateral) right shift by $\tau$ on $L_{\text {loc }}^{2}([0, \infty) ; W)$, and $\mathbf{S}_{\tau}^{*}$ is the left shift by $\tau$ on the same space. (If we restrict $\mathbf{S}_{\tau}$ and $\mathbf{S}_{\tau}^{*}$ from $L_{\text {loc }}^{2}$ to $L^{2}$, then they are adjoint to each other.) For any $u, v \in L_{\text {loc }}^{2}([0, \infty) ; W)$ and any $\tau \geq 0$, the $\tau$-concatenation of $u$ and $v$, denoted 
$u \diamond_{\tau} v$, is the function defined by

$$
u \diamond_{\tau} v=\mathbf{P}_{\tau} u+\mathbf{S}_{\tau} v
$$

Thus, $(u \underset{\tau}{\diamond} v)(t)=u(t)$ for $t \in[0, \tau)$, while $\left(u \diamond_{\tau}^{\diamond} v\right)(t)=v(t-\tau)$ for $t \geq \tau$.

We now recall the precise definition of a well-posed linear system. In Part I, 42 ] and other earlier papers, the term "abstract linear system" was used instead, but it seems that "well-posed" is much more to the point than "abstract", and this is currently the accepted terminology.

Definition 2.2. (This is following Definition I.2.1.) Let $U, X$ and $Y$ be Hilbert spaces and denote $\mathcal{U}=L^{2}([0, \infty) ; U), \mathcal{Y}=L^{2}([0, \infty) ; Y)$. A well-posed linear system on $\mathcal{U}, X$ and $\mathcal{Y}$ is a quadruple $\Sigma=(\mathbb{T}, \Phi, \Psi, \mathbb{F})$, where

(i) $\mathbb{T}=\left(\mathbb{T}_{t}\right)_{t \geq 0}$ is a strongly continuous semigroup of linear operators on $X$,

(ii) $\Phi=\left(\Phi_{t}\right)_{t \geq 0}$ is a family of bounded linear operators from $\mathcal{U}$ to $X$ such that

$$
\Phi_{\tau+t}\left(\underset{\tau}{\diamond v)}=\mathbb{T}_{t} \Phi_{\tau} u+\Phi_{t} v\right.
$$

for every $u, v \in \mathcal{U}$ and all $\tau, t \geq 0$,

(iii) $\Psi=\left(\Psi_{t}\right)_{t \geq 0}$ is a family of bounded linear operators from $X$ to $\mathcal{Y}$ such that

$$
\Psi_{\tau+t} x_{0}=\Psi_{\tau} x_{0} \underset{\tau}{\diamond} \Psi_{t} \mathbb{T}_{\tau} x_{0}
$$

for every $x_{0} \in X$ and all $\tau, t \geq 0$, and $\Psi_{0}=0$,

(iv) $\mathbb{F}=\left(\mathbb{F}_{t}\right)_{t \geq 0}$ is a family of bounded linear operators from $\mathcal{U}$ to $\mathcal{Y}$ such that

$$
\mathbb{F}_{\tau+t}\left(u \diamond_{\tau}^{\diamond v)}=\mathbb{F}_{\tau} u \diamond_{\tau}\left(\Psi_{t} \Phi_{\tau} u+\mathbb{F}_{t} v\right)\right.
$$

for every $u, v \in \mathcal{U}$ and all $\tau, t \geq 0$, and $\mathbb{F}_{0}=0$.

We call $U$ the input space, $X$ the state space and $Y$ the output space of $\Sigma$. The operators $\Phi_{\tau}$ are called input maps, the operators $\Psi_{\tau}$ are called output maps, and the operators $\mathbb{F}_{\tau}$ are called input-output maps.

It follows from (2.1) (with $t=0$ and $v=0$ ) that $\Phi$ is causal, i.e., the present state does not depend on the future input: for all $\tau \geq 0$,

$$
\Phi_{\tau} \mathbf{P}_{\tau}=\Phi_{\tau}
$$

in particular $\Phi_{0}=0$. It follows from the formulas (2.1)-(2.4) that for all $\tau, t \geq 0$,

$$
\Phi_{\tau+t} \mathbf{P}_{\tau}=\mathbb{T}_{t} \Phi_{\tau}, \quad \mathbf{P}_{\tau} \Psi_{\tau+t}=\Psi_{\tau}, \quad \mathbf{P}_{\tau} \mathbb{F}_{\tau+t} \mathbf{P}_{\tau}=\mathbf{P}_{\tau} \mathbb{F}_{\tau+t}=\mathbb{F}_{\tau},
$$

and hence $\mathbf{P}_{\tau} \mathbb{F}_{\tau+t} \mathbf{P}_{[\tau, \tau+t]}=0$. The last identity tells us that $\mathbb{F}$ is causal (i.e., the past output does not depend on the future input).

We now recall some less immediate consequences of Definition 2.2. For the remainder of this section, we use the assumptions of Definition 2.2

Denote the generator of $\mathbb{T}$ by $A$. The space $X_{1}$ is defined as $\mathcal{D}(A)$ with the norm $\left\|z_{1}\right\|=\|(\beta I-A) z\|$, where $\beta \in \rho(A)$, and $X_{-1}$ is the completion of $X$ with respect to the norm $\|z\|_{-1}=\left\|(\beta I-A)^{-1} z\right\|$. The choice of $\beta$ is not important, since different choices lead to equivalent norms on $X_{1}$ and on $X_{-1}$. It is easy to see that $\|\cdot\|_{1}$ is equivalent to the graph norm and $X_{-1}$ is isomorphic to the dual of $\mathcal{D}\left(A^{*}\right)$ with respect to the pivot space $X$. $\mathbb{T}$ can be extended to a semigroup on $X_{-1}$; the generator of the extended semigroup is an extension of $A$ and it has $X$ as its domain. We denote the extensions of $\mathbb{T}_{t}$ and of $A$ by the same symbols. 
It follows from assumptions (i) and (ii) in the definition that there exists a unique $B \in \mathcal{L}\left(U ; X_{-1}\right)$, called the control operator of $\Sigma$, such that for all $t \geq 0$,

$$
\Phi_{t} u=\int_{0}^{t} \mathbb{T}_{t-\sigma} B u(\sigma) \mathrm{d} \sigma
$$

see [29], [38]. The function $\Phi_{t} u$ depends continuously on $t$. The fact that $\Phi_{t} u \in X$ means that $B$ is an admissible control operator for $\mathbb{T}$. Admissible control operators are a subspace of $\mathcal{L}\left(U ; X_{-1}\right)$; we refer to [4], [9], [36], [38] and [43 for investigations of these operators. $B$ is called bounded if $B \in \mathcal{L}(U, X)$, and unbounded otherwise.

Using the identity $\mathbf{P}_{\tau} \Psi_{\tau+t}=\Psi_{\tau}$, we define the operator

$$
\Psi_{\infty}: X \rightarrow L_{\mathrm{loc}}^{2}([0, \infty) ; Y)
$$

by $\Psi_{\infty} x_{0}=\lim _{t \rightarrow \infty} \Psi_{t} x_{0}$. Then $\Psi_{\infty}$ satisfies $\mathbf{P}_{\tau} \Psi_{\infty}=\Psi_{\tau}$ for all $\tau \geq 0 . \Psi_{\infty}$ is called the extended output map of $\Sigma$. By letting $t \rightarrow \infty$ in (2.2), we get

$$
\Psi_{\infty} x_{0}=\Psi_{\infty} x_{0} \diamond_{\tau} \Psi_{\infty} \mathbb{T}_{\tau} x_{0}
$$

for every $x_{0} \in X$ and all $\tau \geq 0$. More generally, any continuous linear operator $\Psi_{\infty}: X \rightarrow L_{\mathrm{loc}}^{2}([0, \infty) ; Y)$ which satisfies (2.7) for every $x_{0} \in X$ and all $\tau \geq 0$ is called an extended output map for $\mathbb{T}$. For every such $\Psi_{\infty}$ there exists a unique $C \in \mathcal{L}\left(X_{1} ; Y\right)$, called the observation operator of $\Psi_{\infty}$ (or of $\Sigma$ ), such that

$$
\left(\Psi_{\infty} x_{0}\right)(t)=C \mathbb{T}_{t} x_{0}
$$

for every $x_{0} \in X_{1}$ and all $t \geq 0$. This determines $\Psi_{\infty}$, since $X_{1}$ is dense in $X$. The Laplace transform of $\Psi_{\infty} x_{0}$ is $C(s I-A)^{-1} x_{0}$, for every $x_{0} \in X$.

An operator $C \in \mathcal{L}\left(X_{1} ; Y\right)$ is called an admissible observation operator for $\mathbb{T}$ if the estimate

$$
\int_{0}^{\tau}\left\|C \mathbb{T}_{t} x_{0}\right\|^{2} \mathrm{~d} t \leq k\left\|x_{0}\right\|^{2}
$$

holds for some $\tau>0$ and for every $x_{0} \in \mathcal{D}(A)$. For further details about such operators we refer to [5, [9, [36, 39] and 43]. It is clear that if $C$ is the observation operator of a well-posed linear system, then $C$ is admissible. $C$ is called bounded if it can be extended to $C \in \mathcal{L}(X ; Y)$, and unbounded otherwise.

For any $C \in \mathcal{L}\left(X_{1}, Y\right)$ we define its $\Lambda$-extension $C_{\Lambda}$ by

$$
C_{\Lambda} x_{0}=\lim _{\lambda \rightarrow+\infty} C \lambda(\lambda I-A)^{-1} x_{0} .
$$

Its domain $\mathcal{D}\left(C_{\Lambda}\right)$ consists of all $x_{0} \in X$ for which the above limit exists.

Proposition 2.3. If $C$ is the observation operator of $\Psi_{\infty}$, then for every $x_{0} \in X$ and for almost every $t \geq 0$ we have that $\mathbb{T}_{t} x_{0} \in \mathcal{D}\left(C_{\Lambda}\right)$ and

$$
\left(\Psi_{\infty} x_{0}\right)(t)=C_{\Lambda} \mathbb{T}_{t} x_{0} .
$$

This extension of (2.8) and related results were derived in 39 .

Using the identity $\mathbf{P}_{\tau} \mathbb{F}_{\tau+t}=\mathbb{F}_{\tau}$, we define the operator $\mathbb{F}_{\infty}: L_{\text {loc }}^{2}([0, \infty) ; U) \rightarrow$ $L_{\text {loc }}^{2}([0, \infty) ; Y)$ by $\mathbb{F}_{\infty} u=\lim _{t \rightarrow \infty} \mathbb{F}_{t} u$. Then $\mathbf{P}_{\tau} \mathbb{F}_{\infty}=\mathbb{F}_{\tau}$ for all $\tau \geq 0$. $\mathbb{F}_{\infty}$ is called the extended input-output map of $\Sigma$. By letting $t \rightarrow \infty$ in (2.3), we get

$$
\mathbb{F}_{\infty}(u \underset{\tau}{\diamond v})=\mathbb{F}_{\infty} u \diamond_{\tau}\left(\Psi_{\infty} \Phi_{\tau} u+\mathbb{F}_{\infty} v\right),
$$

for every $u, v \in \mathcal{U}$ and all $\tau \geq 0$. Taking $u=0$ in 2.11) we get that

$$
\mathbb{F}_{\infty} \mathbf{S}_{\tau}=\mathbf{S}_{\tau} \mathbb{F}_{\infty}
$$


for every $\tau \geq 0$. Any continuous operator $\mathbb{F}_{\infty}: L_{\text {loc }}^{2}([0, \infty) ; U) \rightarrow L_{\text {loc }}^{2}([0, \infty) ; Y)$ which satisfies (2.12) is called shift-invariant or time-invariant. Under mild assumptions, such operators can be represented by transfer functions, see Section I.3. Every shift-invariant operator is causal, which means that for all $\tau \geq 0$,

$$
\mathbf{P}_{\tau} \mathbb{F}_{\infty}=\mathbf{P}_{\tau} \mathbb{F}_{\infty} \mathbf{P}_{\tau}
$$

Definition 2.4. For any $x_{0} \in X$ and any $u \in L_{\text {loc }}^{2}([0, \infty) ; U)$, the state trajectory $x:[0, \infty) \rightarrow X$ and the output function $y \in L_{\mathrm{loc}}^{2}([0, \infty) ; Y)$ of $\Sigma$ corresponding to the initial state $x_{0}$ and the input function $u$ are defined by

$$
\begin{aligned}
x(t) & =\mathbb{T}_{t} x_{0}+\Phi_{t} u, \quad t \geq 0, \\
y & =\Psi_{\infty} x_{0}+\mathbb{F}_{\infty} u .
\end{aligned}
$$

From here we can recover (1.5) (which refers to the finite-dimensional case but remains unchanged in the general well-posed case) by taking $t=\tau$, applying $\mathbf{P}_{\tau}$ to the second equation in (2.14), and using (2.13).

Notation 2.5. For any Hilbert space $W$, any interval $J$ and any $\omega \in \mathbb{R}$ we put

$$
L_{\omega}^{2}(J ; W)=e_{\omega} L^{2}(J ; W),
$$

where $\left(e_{\omega} v\right)(t)=e^{\omega t} v(t)$, with the norm $\left\|e_{\omega} v\right\|_{L_{\omega}^{2}}=\|v\|_{L^{2}}$. We denote by $H^{1}(J ; W)$ the subspace of $L^{2}(J ; W)$ which consists of continuous functions $v$ for which there exists a fuction $\dot{v} \in L^{2}(J ; W)$, called the derivative of $v$, such that

$$
v(b)-v(a)=\int_{a}^{b} \dot{v}(\sigma) \mathrm{d} \sigma,
$$

for all $a, b \in J$. The definition of $H_{\mathrm{loc}}^{1}(J ; W)$ is similar, replacing everywhere $L^{2}(J ; W)$ by $L_{\text {loc }}^{2}(J ; W)$. We introduce a weighted version of $H^{1}(J ; W)$ :

$$
H_{\omega}^{1}(J ; W)=e_{\omega} H^{1}(J ; W) .
$$

We denote by $\mathbb{C}_{\omega}$ the half-plane of all $s \in \mathbb{C}$ with $\operatorname{Re} s>\omega$. The growth bound of the operator semigroup $\mathbb{T}$ with generator $A$ is denoted by $\omega_{\mathbb{T}}$. Thus,

$$
\omega_{\mathbb{T}}=\lim _{t \rightarrow \infty} \frac{1}{t} \log \left\|\mathbb{T}_{t}\right\|=\inf _{t>0} \frac{1}{t} \log \left\|\mathbb{T}_{t}\right\|,
$$

and $(s I-A)^{-1}$ is uniformly bounded on $\mathbb{C}_{\omega}$ if and only if $\omega>\omega_{\mathbb{T}}$.

Proposition 2.6. For any initial state $x_{0} \in X$ and any input $u \in L_{\mathrm{loc}}^{2}([0, \infty) ; U)$, the state trajectory $x$ defined in (2.14) is the unique strong solution in $X_{-1}$ of

$$
\begin{aligned}
& \dot{x}(t)=A x(t)+B u(t), \quad t \geq 0, \\
& x(0)=x_{0} .
\end{aligned}
$$

More precisely, $x$ is continuous with values in $X$, and it is the only function in $H_{\mathrm{loc}}^{1}\left([0, \infty) ; X_{-1}\right)$ with $x(0)=x_{0}$ whose derivative $\dot{x}$ satisfies (2.15) for almost every $t \geq 0$. If moreover $u \in L_{\omega}^{2}([0, \infty) ; U)$ with $\omega>\omega_{\mathbb{T}}$, then $x \in L_{\omega}^{2}([0, \infty) ; X)$ (in addition to being continuous) and its Laplace transform is

$$
\hat{x}(s)=(s I-A)^{-1}\left[x_{0}+B \hat{u}(s)\right], \quad \operatorname{Re} s>\omega .
$$

This result (and also the existence of $B$ ) is contained in [28], [29], [38].

As shown in [39, Proposition 2.3] and Proposition I.4.1, for every $\omega>\omega_{\mathbb{T}}, \Psi_{\infty}$ is bounded from $X$ to $L_{\omega}^{2}([0, \infty) ; Y)$ and $\mathbb{F}_{\infty}$ is bounded from $L_{\omega}^{2}([0, \infty) ; U)$ to $L_{\omega}^{2}([0, \infty) ; Y)$. This enables us to represent also the output function $y$ from (2.14) via its Laplace transform, as shown in the following theorem. 
Theorem 2.7. There exists an analytic $\mathcal{L}(U ; Y)$-valued function $\mathbf{G}$ on $\mathbb{C}_{\omega_{\mathbb{T}}}$, called the transfer function of $\Sigma$, which has the following properties:

(1) For every $x_{0} \in X$ and $u \in L_{\omega}^{2}([0, \infty) ; U)$ with $\omega>\omega_{\mathbb{T}}$, the corresponding output function $y=\Psi_{\infty} x_{0}+\mathbb{F}_{\infty} u$ is in $L_{\omega}^{2}([0, \infty) ; Y)$ and its Laplace transform is

$$
\hat{y}(s)=C(s I-A)^{-1} x_{0}+\mathbf{G}(s) \hat{u}(s), \quad \operatorname{Re} s>\omega .
$$

(2) $\mathbf{G}$ satisfies for all $s, \beta \in \mathbb{C}_{\omega_{\mathbb{T}}}$

$$
\mathbf{G}(s)-\mathbf{G}(\beta)=C\left[(s I-A)^{-1}-(\beta I-A)^{-1}\right] B
$$

(equivalently, $\left.\mathbf{G}^{\prime}(s)=-C(s I-A)^{-2} B\right)$.

(3) $\mathbf{G}$ is bounded on $\mathbb{C}_{\omega}$ for every $\omega>\omega_{\mathbb{T}}$.

Point (1) above was proved in Part I (see Theorems I.3.6, I.4.1 and 39, formula (3.6)]). Point (2) is Remark I.4.10 or [28, formula (2.3)]. For point (3) we refer again to Theorems I.3.6 and I.4.1. Point (2) above shows that $\mathbf{G}$ is determined by $A, B$ and $C$ up to an additive constant operator.

We denote by $\gamma_{\mathbb{F}}$ the infimum of those $\omega \in \mathbb{R}$ for which $\mathbb{F}_{\infty}$ is bounded from $L_{\omega}^{2}([0, \infty) ; U)$ to $L_{\omega}^{2}([0, \infty) ; Y)$. Equivalently, $\gamma_{\mathbb{F}}$ is the infimum of those $\omega \in \mathbb{R}$ for which $\mathbf{G}$ has a bounded analytic continuation to $\mathbb{C}_{\omega}$, see Theorem I.3.6. This $\gamma_{\mathbb{F}} \in$ $[-\infty, \infty)$ is called the growth bound of $\mathbb{F}_{\infty}$. It follows from what we have already said that $\gamma_{\mathbb{F}} \leq \omega_{\mathbb{T}}$ (see also Proposition I.4.1). The analytic continuation of $\mathbf{G}$ to $\mathbb{C}_{\gamma_{\mathrm{F}}}$ does not necessarily satisfy (2.18), see Remark I.4.8. The following extension of (2.17) (with $\left.x_{0}=0\right)$ holds: if $\mathbb{F}_{\infty}$ is bounded from $L_{\omega}^{2}([0, \infty) ; U)$ to $L_{\omega}^{2}([0, \infty) ; Y$ ) (for example, and if $\left.\omega>\gamma_{\mathbb{F}}\right)$, if $u \in L_{\omega}^{2}([0, \infty) ; U)$ and $y=\mathbb{F}_{\infty} u$, then

$$
\hat{y}(s)=\mathbf{G}(s) \hat{u}(s), \quad \operatorname{Re} s>\omega .
$$

It follows that for such $\omega$, the norm of $\mathbb{F}_{\infty}$ from $L_{\omega}^{2}$ to $L_{\omega}^{2}$ is the supremum of $\|\mathbf{G}(s)\|$ over all $s \in \mathbb{C}_{\omega}$ (see again Theorem I.3.6).

\section{The operators $C \& D$ AND $S_{\Sigma}(s)$}

In this section we introduce the system operator $S_{\Sigma}(s)$ of a well-posed linear system $\Sigma$, which we have encountered in (1.2) for the finite-dimensional case. The "second row" of this operator is the combined observation/feedthrough operator $C \& D$, which in the finite-dimensional case would be the matrix $\left[\begin{array}{ll}C & D\end{array}\right]$. This operator has appeared (somewhat implicitly) in Salamon [28], and according to Arov and Nudelman [1], it was introduced by Yu. L. Shmulyan in 1986. Theorems 3.1 and 3.2 give a representation for the state trajectory and the output function of a well-posed linear system, in terms of $S_{\Sigma}(0)$ and an extension of $C \& D$. These theorems give the infinite-dimensional version of the equations (1.1) and (1.3) in full generality. We also show that $C \& D$ can be split as $C \& D=[\bar{C} D]$, where $\bar{C}$ is one of many possible extensions of $C$ to a larger space than $\mathcal{D}(A)$ and $D$ is a bounded operator that depends on the extension $\bar{C}$ (see Corollary 3.5 ).

Throughout this section, we assume that $\Sigma=(\mathbb{T}, \Phi, \Psi, \mathbb{F})$ is a well-posed linear system with input space $U$, state space $X$ and output space $Y$. We denote by $A$ the generator of $\mathbb{T}$, by $B$ the control operator of $\Sigma$, by $C$ its observation operator and by $\mathbf{G}$ its transfer function. We use the notation introduced in Section 2, such as $\mathbf{P}_{\tau}, \Psi_{\infty}, \mathbb{F}_{\infty}, X_{1}, X_{-1}, \omega_{\mathbb{T}}, C_{\Lambda}, \mathbf{S}_{\tau}^{*}, L_{\omega}^{2}(J ; W), H_{\mathrm{loc}}^{1}(J ; W), H_{\omega}^{1}(J ; W)$ and $\mathbb{C}_{\omega}$. 
We introduce the dense subspace $V$ of $X \times U$ defined by

$$
V=\left\{\left[\begin{array}{l}
x_{0} \\
u_{0}
\end{array}\right] \in X \times U \mid A x_{0}+B u_{0} \in X\right\} .
$$

(To see that $V$ is indeed dense, take an arbitrary pair $\left(x_{0}, u_{0}\right) \in X \times U$, take $\lambda \in \rho(A)$, denote $z_{0}=(A-\lambda I) x_{0}+B u_{0}$, take a sequence $\left(z_{n}\right)$ in $X$ such that $z_{n} \rightarrow z_{0}$ (in $X_{-1}$ ) and define $x_{n}$ by the equation $z_{n}=(A-\lambda I) x_{n}+B u_{0}$. Then $\left(x_{n}, u_{0}\right) \in V$ and $x_{n} \rightarrow x_{0}$ (in $\left.X\right)$.) $V$ is a Hilbert space with the norm

$$
\left\|\left[\begin{array}{l}
x_{0} \\
u_{0}
\end{array}\right]\right\|_{V}=\left(\left\|x_{0}\right\|_{X}^{2}+\left\|u_{0}\right\|_{U}^{2}+\left\|A x_{0}+B u_{0}\right\|_{X}^{2}\right)^{1 / 2} .
$$

Note that $[A B] \in \mathcal{L}(V ; X)$. We define the operator $C \& D: V \rightarrow Y$ by

$$
C \& D\left[\begin{array}{l}
x_{0} \\
u_{0}
\end{array}\right]=C\left[x_{0}-(\beta I-A)^{-1} B u_{0}\right]+\mathbf{G}(\beta) u_{0},
$$

where $\beta \in \mathbb{C}_{\omega_{\mathbb{T}}}$ is arbitrary (i.e., the result is independent of $\beta$ as long as $\operatorname{Re} \beta>\omega_{\mathbb{T}}$, as is easy to verify). We call $C \& D$ the combined observation/feedthrough operator of $\Sigma$. In Arov and Nudelman [1, this operator is denoted by $N$. Note that $C \& D \in$ $\mathcal{L}(V, Y)$, because $\left[I-(\beta I-A)^{-1} B\right] \in \mathcal{L}\left(V ; X_{1}\right)$.

We also introduce the system operator of $\Sigma, S_{\Sigma}(s): V \rightarrow X \times Y$, by

$$
S_{\Sigma}(s)=\left[\begin{array}{lr}
A & B \\
C \& D
\end{array}\right]-\left[\begin{array}{cc}
s I & 0 \\
0 & 0
\end{array}\right], \quad \text { for all } s \in \mathbb{C} .
$$

It is clear that $S_{\Sigma}(s)$ is bounded from $V$ to $X \times Y$. $S_{\Sigma}(s)$ can also be interpreted as an unbounded operator from $X \times U$ to $X \times Y$ with dense domain $\mathcal{D}\left(S_{\Sigma}(s)\right)=V$ (independent of $s$ ), and a short argument shows that this unbounded operator is closed. (The argument uses the fact that if $x_{n} \rightarrow x$ in $X, u_{n} \rightarrow u$ in $U$ and $A x_{n}+B u_{n} \rightarrow z$ in $X$, then $\left[\begin{array}{l}x_{n} \\ u_{n}\end{array}\right] \rightarrow\left[\begin{array}{l}x \\ u\end{array}\right]$ in $V$.) If $C$ is bounded, then we denote

$$
D=\mathbf{G}(\beta)-C(\beta I-A)^{-1} B,
$$

where $\operatorname{Re} \beta>\omega_{\mathbb{T}}$ (note that $D$ is independent of $\beta$, as follows from (2.18)). Then we obtain that $C \& D=\left[\begin{array}{ll}C & D\end{array}\right]$ (which is defined on all of $X \times U$ ) and hence

$$
S_{\Sigma}(s)=\left[\begin{array}{cc}
A-s I & B \\
C & D
\end{array}\right]
$$

as in the finite-dimensional case, see (1.2). A similar splitting of $C \& D$ is possible also for systems with an unbounded observation operator $C$, but it requires an extension of $C$ which is not unique, see Corollary 3.5 .

Theorem 3.1. (i) Assume that $u \in H_{\mathrm{loc}}^{1}([0, \infty) ; U)$ and $\left[\begin{array}{c}x_{0} \\ u(0)\end{array}\right] \in V$. The state trajectory $x$ and the output function $y$ are defined as in (2.14). Then

$$
x \in C^{1}([0, \infty) ; X), \quad\left[\begin{array}{l}
x \\
u
\end{array}\right] \in C([0, \infty) ; V), \quad y \in H_{\mathrm{loc}}^{1}([0, \infty) ; Y),
$$

and for every $t \geq 0$ we have that

$$
\left[\begin{array}{l}
\dot{x}(t) \\
y(t)
\end{array}\right]=S_{\Sigma}(0)\left[\begin{array}{l}
x(t) \\
u(t)
\end{array}\right] .
$$

If $u \in H_{\omega}^{1}([0, \infty) ; U)$ with $\omega>\omega_{\mathbb{T}}$, then $y \in H_{\omega}^{1}([0, \infty) ; Y)$. 
(ii) The transfer function $\mathbf{G}$ of $\Sigma$ is given by

$$
\mathbf{G}(s)=C \& D\left[\begin{array}{c}
(s I-A)^{-1} B \\
I
\end{array}\right], \quad \operatorname{Re} s>\omega_{\mathbb{T}} .
$$

This theorem is the infinite-dimensional counterpart of (1.1) and of (1.3). This theorem can be derived from results in [1, [28], or [36], but for completeness we include a proof. (Parts of this proof will be needed later, too.)

Proof. We begin by observing that it suffices to prove (i) in the case when $u \in$ $H_{\omega}^{1}([0, \infty) ; U)$ with $\omega>\omega_{\mathbb{T}}$. This is true since, for all $\tau>0$, the restrictions of $x$ and $y$ to $[0, \tau]$ do not depend on the restriction of $u$ to $[\tau, \infty)$ (by causality), and it is possible to redefine any function $u \in H_{\mathrm{loc}}^{1}([0, \infty) ; U)$ on the interval $[\tau, \infty)$ in such a way that the modified function belongs to $H_{\omega}^{1}([0, \infty) ; U)$.

We denote $\mathcal{U}=L_{\omega}^{2}([0, \infty) ; U)$. For every $t \geq 0$, on $X \times \mathcal{U}$ we define the bounded operator $\mathfrak{G}_{t}$ by

$$
\mathfrak{G}_{t}=\left[\begin{array}{cc}
\mathbb{T}_{t} & \Phi_{t} \\
0 & \mathbf{S}_{t}^{*}
\end{array}\right]
$$

Then $\mathfrak{G}=\left(\mathfrak{G}_{t}\right)_{t \geq 0}$ is a strongly continuous semigroup, whose growth bound is $\omega$. (Such semigroups were used in Grabowski and Callier [5] to study admissibility. More elaborate semigroups of this kind will appear in Section [6]) The generator of this semigroup is

$$
\mathcal{A}=\left[\begin{array}{cc}
A & B \delta_{0} \\
0 & \frac{\mathrm{d}}{\mathrm{d} \xi}
\end{array}\right]
$$

where $\delta_{0} u=u(0)$ and

$$
\mathcal{D}(\mathcal{A})=\left\{\left[\begin{array}{c}
x_{0} \\
u
\end{array}\right] \in X \times H_{\omega}^{1}([0, \infty) ; U) \mid\left[\begin{array}{c}
x_{0} \\
u(0)
\end{array}\right] \in V\right\} .
$$

$\mathcal{D}(\mathcal{A})$ is a Hilbert space with the norm

$$
\left\|\left[\begin{array}{c}
x_{0} \\
u
\end{array}\right]\right\|=\left\|(\beta I-\mathcal{A})\left[\begin{array}{c}
x_{0} \\
u
\end{array}\right]\right\|_{X \times \mathcal{U}}
$$

(this is like the space $X_{1}$ encountered earlier). The conditions in part (i) of the theorem mean that $\left[\begin{array}{c}x_{0} \\ u\end{array}\right] \in \mathcal{D}(\mathcal{A})$. It follows that the function $z$ defined by $z(t)=$ $\mathfrak{G}_{t}\left[\begin{array}{c}x_{0} \\ u\end{array}\right]$ is of class $C^{1}$ with values in $X \times \mathcal{U}$, which implies that $x$ (the first component of $z$ ) is of class $C^{1}$ with values in $X$, as claimed in part (i). The fact that $\left[\begin{array}{c}x_{0} \\ u\end{array}\right] \in$ $\mathcal{D}(\mathcal{A})$ also implies that $z$ is continuous with values in $\mathcal{D}(\mathcal{A})$, which implies that the function $\left[\begin{array}{l}x \\ u\end{array}\right]=\left[\begin{array}{ll}I & 0 \\ 0 & \delta_{0}\end{array}\right] z$ is continuous with values in $V$, as claimed in part (i).

We define the operator $\mathcal{C}: \mathcal{D}(\mathcal{A}) \rightarrow Y$ by $\mathcal{C}=C \& D\left[\begin{array}{ll}I & 0 \\ 0 & \delta_{0}\end{array}\right]$, i.e.,

$$
\mathcal{C}\left[\begin{array}{c}
x_{0} \\
u
\end{array}\right]=C \& D\left[\begin{array}{c}
x_{0} \\
u(0)
\end{array}\right]
$$

It follows from the continuity of $C \& D$ on $V$ that $\mathcal{C}$ is bounded from $\mathcal{D}(\mathcal{A})$ to $Y$. Now we show that $\mathcal{C}$ is an admissible observation operator for $\mathfrak{G}$. For $\left[\begin{array}{c}x_{0} \\ u\end{array}\right] \in \mathcal{D}(\mathcal{A})$, define the function $y$ for $t \geq 0$ by

$$
y(t)=\mathcal{C} \mathfrak{G}_{t}\left[\begin{array}{c}
x_{0} \\
u
\end{array}\right]=C \& D\left[\begin{array}{l}
x(t) \\
u(t)
\end{array}\right]
$$


so that obviously $y \in C([0, \infty) ; Y)$ and $e^{-\omega t} y(t)$ is bounded. Hence, $y$ has a Laplace transform $\hat{y}$ defined on $\mathbb{C}_{\omega}$ and, by (2.16) and (3.2) with $\beta=s$,

$$
\begin{aligned}
\hat{y}(s) & =C \& D\left[\begin{array}{l}
\hat{x}(s) \\
\hat{u}(s)
\end{array}\right] \\
& =C \& D\left[\begin{array}{c}
(s I-A)^{-1}\left[x_{0}+B \hat{u}(s)\right] \\
\hat{u}(s)
\end{array}\right] \\
& =C(s I-A)^{-1} x_{0}+\mathbf{G}(s) \hat{u}(s) .
\end{aligned}
$$

Comparing this with (2.17), we conclude that $y$ is the output function of $\Sigma$. Hence, the lower half of (3.3) holds. The upper half of (3.3) is a direct consequence of Proposition 2.6. By the well-posedness of $\Sigma$,

$$
\|y\|_{L_{\omega}^{2}} \leq k\left\|\left[\begin{array}{c}
x_{0} \\
u
\end{array}\right]\right\|_{X \times \mathcal{U}}
$$

This implies that $\mathcal{C}$ is admissible for the semigroup $\mathfrak{G}$.

It follows from the admissibility of $\mathcal{C}$ that for $\left[\begin{array}{c}x_{0} \\ u\end{array}\right] \in \mathcal{D}(\mathcal{A})$, the output function $y$ of $\Sigma$, which is given by (3.5), is in $H_{\omega}^{1}([0, \infty) ; Y)$. Indeed, its derivative is given, for almost every $t \geq 0$, by

$$
\dot{y}(t)=\mathcal{C}_{\Lambda} \mathfrak{G}_{t} \mathcal{A}\left[\begin{array}{c}
x_{0} \\
u
\end{array}\right]
$$

and this function is in $L_{\omega}^{2}([0, \infty) ; Y)$, because it is of the same structure as the function in (2.10), with $\mathcal{C}$ in place of $C, \mathfrak{G}$ in place of $\mathbb{T}$ and $\mathcal{A}\left[\begin{array}{c}x_{0} \\ u\end{array}\right]$ in place of $x_{0}$ (see the comments before Theorem 2.7). This completes the proof of part (i). Part (ii) is an easy consequence of (2.18) and (3.2).

We define the $\Lambda$-extension of $C \& D$ by

$$
[C \& D]_{\Lambda}\left[\begin{array}{l}
x_{0} \\
u_{0}
\end{array}\right]=C_{\Lambda}\left[x_{0}-(\beta I-A)^{-1} B u_{0}\right]+\mathbf{G}(\beta) u_{0}
$$

where $\beta \in \mathbb{C}_{\omega_{\mathbb{T}}}$ is arbitrary. Its domain $\mathcal{D}\left([C \& D]_{\Lambda}\right)$ consists of those $\left[\begin{array}{l}x_{0} \\ u_{0}\end{array}\right] \in X \times U$ for which $x_{0}-(\beta I-A)^{-1} B u_{0} \in \mathcal{D}\left(C_{\Lambda}\right)$. The following result was stated in [40] (without using the notation $C \& D$ ), but so far no proof has been published.

Theorem 3.2. For $x_{0} \in X$ and $u \in L_{\mathrm{loc}}^{2}([0, \infty) ; U)$, let the state trajectory $x$ and the output function $y$ be defined as in (2.14). Then for almost every $t \geq 0$ we have $\left[\begin{array}{l}x(t) \\ u(t)\end{array}\right] \in \mathcal{D}\left([C \& D]_{\Lambda}\right)$ and

$$
y(t)=[C \& D]_{\Lambda}\left[\begin{array}{l}
x(t) \\
u(t)
\end{array}\right]
$$

A small clarification may be needed here: an element in $L_{\text {loc }}^{2}([0, \infty) ; U)$ or in $L_{\text {loc }}^{2}([0, \infty) ; Y)$ is an equivalence class of functions that are equal almost everywhere, so that for a specific $t \geq 0, u(t)$ and $y(t)$ are not defined. However, the theorem holds for any choice of functions in the equivalence classes of $u$ and $y$.

In order to prove Theorem 3.2, we need the following lemma.

Lemma 3.3. We use the notation $\omega, \mathcal{U}, \mathfrak{G}, \mathcal{A}$ and $\mathcal{C}$ from the proof of Theorem 3.1, and $\mathcal{C}_{\Lambda}$ denotes the $\Lambda$-extension of $\mathcal{C}$, defined in the usual way (see (2.9) using the generator $\mathcal{A}$. If $\left[\begin{array}{c}z_{0} \\ v\end{array}\right] \in \mathcal{D}\left(\mathcal{C}_{\Lambda}\right)$ is such that the limit

$$
\lim _{\lambda \rightarrow+\infty} \lambda \hat{v}(\lambda)=v_{0}
$$


exists in $U$, then $\left[\begin{array}{l}z_{0} \\ v_{0}\end{array}\right] \in \mathcal{D}\left([C \& D]_{\Lambda}\right)$ and

$$
\mathcal{C}_{\Lambda}\left[\begin{array}{c}
z_{0} \\
v
\end{array}\right]=[C \& D]_{\Lambda}\left[\begin{array}{c}
z_{0} \\
v_{0}
\end{array}\right] .
$$

This lemma justifies the name " $\Lambda$-extension" used for $[C \& D]_{\Lambda}$, even though $C \& D$ is not an observation operator.

Proof. Recall from the proof of Theorem 3.1 that for $\left[\begin{array}{c}z_{0} \\ v\end{array}\right] \in \mathcal{D}(\mathcal{A})$, the function $w$ defined for $t \geq 0$ by $w(t)=\mathcal{C} \mathfrak{G}_{t}\left[\begin{array}{c}z_{0} \\ v\end{array}\right]$ is the output function of $\Sigma$ corresponding to the initial state $z_{0}$ and the input function $v$, so that $\hat{w}$ is given by $\hat{w}(s)=$ $C(s I-A)^{-1} z_{0}+\mathbf{G}(s) \hat{v}(s)$, see 2.17). On the other hand, computing $\hat{w}$ directly from the definition of $w$, we get an expression in terms of $\mathcal{C}$ and $\mathcal{A}$, and the two expressions for $\hat{w}$ must be equal:

$$
\mathcal{C}(s I-\mathcal{A})^{-1}\left[\begin{array}{c}
z_{0} \\
v
\end{array}\right]=C(s I-A)^{-1} z_{0}+\mathbf{G}(s) \hat{v}(s),
$$

for all $s \in \mathbb{C}_{\omega}$. By continuous extension, this formula remains valid for all $\left[\begin{array}{c}z_{0} \\ v\end{array}\right] \in$ $X \times \mathcal{U}$. From this formula and (2.18) we deduce that for large $\lambda>0$

$$
\begin{aligned}
& \mathcal{C} \lambda(\lambda I-\mathcal{A})^{-1}\left[\begin{array}{c}
z_{0} \\
v
\end{array}\right]=C \lambda(\lambda I-A)^{-1}\left[z_{0}-(\beta I-A)^{-1} B v_{0}\right] \\
&+\lambda C(\lambda I-A)^{-1}(\beta I-A)^{-1} B v_{0}+\mathbf{G}(\lambda) \lambda \hat{v}(\lambda) \\
&=C \lambda(\lambda I-A)^{-1}\left[z_{0}-(\beta I-A)^{-1} B v_{0}\right]+\frac{\lambda}{\lambda-\beta} \mathbf{G}(\beta) v_{0} \\
&+\mathbf{G}(\lambda)\left[\lambda \hat{v}(\lambda)-\frac{\lambda}{\lambda-\beta} v_{0}\right] .
\end{aligned}
$$

If $\left[\begin{array}{c}z_{0} \\ v\end{array}\right] \in \mathcal{D}\left(\mathcal{C}_{\Lambda}\right)$, then the above expression must have a limit as $\lambda \rightarrow+\infty$. If moreover (3.8) holds, then the last term tends to zero. Hence, taking limits as $\lambda \rightarrow+\infty$, we obtain that $z_{0}-(\beta I-A)^{-1} B v_{0} \in \mathcal{D}\left(C_{\Lambda}\right)$ and the formula (3.9) holds.

Proof of Theorem 3.2. We use again the notation $\omega, \mathcal{U}, \mathfrak{G}, \mathcal{A}$ and $\mathcal{C}$ from the proof of Theorem 3.1 (recall that $\omega>\omega_{\mathbb{T}}$ ). First we assume that $u \in \mathcal{U}$. Then we know from Theorem 2.7 that $y \in L_{\omega}^{2}([0, \infty) ; Y)$. According to (2.17) and (3.10),

$$
\hat{y}(s)=\mathcal{C}(s I-\mathcal{A})^{-1}\left[\begin{array}{c}
x_{0} \\
u
\end{array}\right], \quad \operatorname{Re} s>\omega .
$$

Thus, $y$ is the output generated by $\mathcal{C}$ and $\mathfrak{G}$ via (3.5) and continuous extension to all of $X \times \mathcal{U}$. According to Proposition 2.3 , we have for almost every $t \geq 0$ that

$$
\left[\begin{array}{c}
x(t) \\
\mathbf{S}_{t}^{*} u
\end{array}\right]=\mathfrak{G}_{t}\left[\begin{array}{c}
x_{0} \\
u
\end{array}\right] \in \mathcal{D}\left(\mathcal{C}_{\Lambda}\right) \text { and } y(t)=\mathcal{C}_{\Lambda}\left[\begin{array}{l}
x(t) \\
\mathbf{S}_{t}^{*} u
\end{array}\right]
$$

On the other hand, consider the strongly continuous semigroup $\mathbf{S}^{*}=\left(\mathbf{S}_{t}^{*}\right)_{t \geq 0}$ acting on $\mathcal{U}$, with generator $\frac{\mathrm{d}}{\mathrm{d} \xi}$ and with the admissible observation operator $\delta_{0}$ defined on $\mathcal{D}\left(\frac{\mathrm{d}}{\mathrm{d} \xi}\right)=H_{\omega}^{1}([0, \infty) ; U)$ by $\delta_{0} u=u(0)$. Obviously, for $u \in H_{\omega}^{1}([0, \infty) ; U)$ we have $\delta_{0} \mathbf{S}_{t}^{*} u=u(t)$, so that the extended output map corresponding to $\mathbf{S}^{*}$ and $\delta_{0}$ (as in (2.8) ) is the identity. According to Proposition 2.3. for almost every $t \geq 0$,

$$
\mathbf{S}_{t}^{*} u \in \mathcal{D}\left(\delta_{0 \Lambda}\right) \text { and } u(t)=\delta_{0 \Lambda} \mathbf{S}_{t}^{*} u .
$$


It is easy to see that for any $v \in \mathcal{U}$ we have $v \in \mathcal{D}\left(\delta_{0 \Lambda}\right)$ if and only if $\lim _{\lambda \rightarrow+\infty} \lambda \hat{v}(\lambda)$ exists, and then $\delta_{0 \Lambda} v$ is this limit. Thus, for almost every $t \geq 0$,

$$
u(t)=\lim _{\lambda \rightarrow+\infty} \lambda \widehat{\mathbf{S}_{t}^{*} u}(\lambda) .
$$

This fact, together with (3.11), shows (by denoting $z_{0}=x(t), v=\mathbf{S}_{t}^{*} u$ and $v_{0}=$ $u(t))$ that for almost every $t \geq 0,\left[\begin{array}{l}x(t) \\ \mathbf{S}_{t}^{*} u\end{array}\right]$ satisfies the conditions of Lemma 3.3 According to this lemma, we obtain that (3.7) holds almost everywhere.

Now if we have an arbitrary $u \in L_{\mathrm{loc}}^{2}([0, \infty) ; U)$, then on any finite time interval $[0, \tau]$, the conclusion of the theorem holds. This is because we can replace $u$ by $\mathbf{P}_{\tau} u$, which is in $\mathcal{U}$, and by the causality of $\Sigma$ neither $x$ nor $y$ will change on $[0, \tau]$. This implies that the conclusion of the theorem holds on the whole interval $[0, \infty)$.

Now we address the question of splitting the operator $C \& D$ in a way similar to the case of a bounded $C$, discussed before Theorem 3.1 For some time it has been considered an open question among specialists whether such a splitting is possible in general. We give here an affirmative answer, but the splitting is not unique, and we cannot point to a "privileged" or "best" way to split $C \& D$. If the system is regular, as defined in Part I, then of course we have a privileged splitting - this will be discussed (among other things) in the next section.

To discuss splittings of $C \& D$, we need the following space, which has appeared often in papers dealing with well-posed systems (including Part I):

$$
Z=X_{1}+(\beta I-A)^{-1} B U,
$$

where $\beta \in \rho(A)$ (the space $Z$ does not depend on the choice of $\beta$ ). $Z$ is a Hilbert space with the following factor space norm:

$$
\|z\|_{Z}=\inf _{z=x+(\beta I-A)^{-1} B v}\left(\|x\|_{1}^{2}+\|v\|_{U}^{2}\right)^{\frac{1}{2}} .
$$

It is easy to see that $X_{1} \subset Z \subset X$ with continuous embeddings, but $X_{1}$ need not be dense in $Z$. In fact, if $B U \cap X=\{0\}$ (such a control operator $B$ is called strictly unbounded), then it is easy to see that $X_{1}$ is a closed subspace of $Z$.

Theorem 3.4. With the space $Z$ defined as above, the operator $C$ can be extended to an operator $\bar{C} \in \mathcal{L}(Z ; Y)$.

Proof. The first (and main) step is to show that $C$ is continuous on $\mathcal{D}(A)$ endowed with the norm of $Z$. In other words, we show that if the sequence $\left(z_{n}\right)$ in $\mathcal{D}(A)$ is such that $\left\|z_{n}\right\|_{Z} \rightarrow 0$, then $\left\|C z_{n}\right\|_{Y} \rightarrow 0$. If the sequence $\left(z_{n}\right)$ is as above, then we can find sequences $\left(x_{n}\right)$ in $\mathcal{D}(A)=X_{1}$ and $\left(v_{n}\right)$ in $U$ such that

$$
z_{n}=x_{n}+(\beta I-A)^{-1} B v_{n}, \quad\left\|x_{n}\right\|_{1} \rightarrow 0, \quad\left\|v_{n}\right\|_{U} \rightarrow 0 .
$$

Since both $z_{n}$ and $x_{n}$ are in $\mathcal{D}(A)$, so is $(\beta I-A)^{-1} B v_{n}$, so that $B v_{n} \in X$.

Consider the system $\Sigma_{n}$ obtained from $\Sigma$ by restricting the input to scalar multiples of $v_{n}$. The control operator of $\Sigma_{n}$ is $B v_{n}$ and its transfer function is $\mathbf{G}(s) v_{n}$. Clearly $\Sigma_{n}$ is regular in the sense of Part I, so that by Theorem I.4.7,

$$
\mathbf{G}(s) v_{n}=C(s I-A)^{-1} B v_{n}+D_{n},
$$

where $D_{n} \in Y$ is given, according to Theorem I.5.8, by

$$
D_{n}=\lim _{\lambda \rightarrow+\infty} \mathbf{G}(\lambda) v_{n} .
$$


Choose $\omega>\omega_{\mathbb{T}}$, so that $\mathbf{G}$ is bounded on $\mathbb{C}_{\omega}$, and denote

$$
\|\mathbf{G}\|_{\omega}=\sup _{s \in \mathbb{C}_{\omega}}\|\mathbf{G}(s)\|
$$

Then it is clear from the formula for $D_{n}$ that $\left\|D_{n}\right\|_{Y} \leq\|\mathbf{G}\|_{\omega}\left\|v_{n}\right\|_{U}$. Now it follows from (3.13) that

$$
\left\|C(s I-A)^{-1} B v_{n}\right\|_{Y} \leq 2\|\mathbf{G}\|_{\omega}\left\|v_{n}\right\|_{U}
$$

for all $s \in \mathbb{C}_{\omega}$ and for all $n \in \mathbb{N}$. Assume without loss of generality that $\beta \in$ $\mathbb{C}_{\omega}$. Then the above estimate shows that $\left\|C(\beta I-A)^{-1} B v_{n}\right\|_{Y} \rightarrow 0$. Since clearly $\left\|C x_{n}\right\|_{Y} \rightarrow 0$, we see from the decomposition in 3.12 that $\left\|C z_{n}\right\|_{Y} \rightarrow 0$.

Let us denote by $Z_{1}$ the closure of $\mathcal{D}(A)$ in $Z$. The continuity of $C$ on $\mathcal{D}(A)$ with the norm of $Z$ implies that $C$ has a unique continuous extension to $Z_{1}$, and we denote this extension also by $C$. Let $Z_{2}$ denote the orthogonal complement of $Z_{1}$ in $Z$, so that any $z \in Z$ has a unique decomposition $z=z_{1}+z_{2}$, with $z_{1} \in Z_{1}$ and $z_{2} \in Z_{2}$. For any $E \in \mathcal{L}\left(Z_{2} ; Y\right)$ we can define the desired extension $\bar{C}$ by $\bar{C}\left(z_{1}+z_{2}\right)=C z_{1}+E z_{2}$.

The above proof shows clearly that unless $\mathcal{D}(A)$ is dense in $Z$, the extension $\bar{C}$ is not unique; it depends on the arbitrary bounded operator $E$.

Corollary 3.5. With the notation of Theorem 3.4, take $\beta \in \mathbb{C}$ with $\operatorname{Re} \beta>\omega_{\mathbb{T}}$ and define $D \in \mathcal{L}(U ; Y)$ by

$$
D=\mathbf{G}(\beta)-\bar{C}(\beta I-A)^{-1} B
$$

Then $D$ is independent of the choice of $\beta$, and the operator $C \& D$ can be extended to an operator $\overline{C \& D} \in \mathcal{L}(Z \times U ; Y)$ as follows:

$$
\overline{C \& D}\left[\begin{array}{l}
x_{0} \\
u_{0}
\end{array}\right]=\bar{C} x_{0}+D u_{0}
$$

The proof of this corollary is easy, and we omit it. Note that $D$ depends on $\bar{C}$. It is clear how to write $S_{\Sigma}$ using $\bar{C}$ and $D$. The formula (3.4) simplifies to

$$
\mathbf{G}(s)=\bar{C}(s I-A)^{-1} B+D, \quad \operatorname{Re} s>\omega_{\mathbb{T}} .
$$

Finally, we give some factorizations of $S_{\Sigma}(s)$. In the following proposition, we denote $\mathbf{G}(s)=C \& D\left[\begin{array}{c}(s I-A)^{-1} B \\ I\end{array}\right]$, for all $s \in \rho(A)$. For $\operatorname{Re} s>\omega_{\mathbb{T}}$ this is the usual expression for the transfer function, see (3.4), but now we need also points $s$ with $\operatorname{Re} s \leq \omega_{\mathbb{T}}$. The analytic function $\mathbf{G}$ defined in this way is not necessarily the analytic continuation of the transfer function, as shown in a counterexample in Remark I.4.8. However, we hope that this notation will not lead to any confusion.

Proposition 3.6. For $s \in \rho(A),\left[\begin{array}{cc}I(A-s I)^{-1} B \\ 0 \\ I\end{array}\right]$ maps $V$ one-to-one onto $X_{1} \times U$. Hence, $\left[\begin{array}{cc}A-s I & B \\ 0 & I\end{array}\right]$ maps $V$ one-to-one onto $X \times U$, and hence the product of the last two factors in (1.4) maps $V$ to $X_{1} \times Y$. For $s \in \rho(A)$, the factorization (1.4) holds, so that $S_{\Sigma}(s)$ is invertible if and only if $\mathbf{G}(s)$ is invertible. Formula (1.4) can be written also in the form

$$
S_{\Sigma}(s)=\left[\begin{array}{cc}
I & 0 \\
C(A-s I)^{-1} & I
\end{array}\right]\left[\begin{array}{cc}
A-s I & 0 \\
0 & \mathbf{G}(s)
\end{array}\right]\left[\begin{array}{cc}
I & (A-s I)^{-1} B \\
0 & I
\end{array}\right] .
$$


Moreover, for every $\lambda \in \mathbb{C}$ and $s \in \rho(A)$, we have

$$
S_{\Sigma}(\lambda)=\left[\begin{array}{cc}
A-\lambda I & (\lambda-s)(A-s I)^{-1} B \\
C & \mathbf{G}(s)
\end{array}\right]\left[\begin{array}{cc}
I & (A-s I)^{-1} B \\
0 & I
\end{array}\right] .
$$

We leave the easy proof of this proposition to the reader (see the definitions of $V, C \& D$ and $S_{\Sigma}(s)$ ). Note that the second factor in (3.15), when regarded as an operator on $X \times U$, tends to the identity (in norm) as $\operatorname{Re} s \rightarrow+\infty$.

\section{WEAKLY REGULAR SYSTEMS}

In this section we introduce weakly regular linear systems following Weiss ${ }^{2}$ [45] and we extend the main results of Part I to weakly regular systems. For a weakly regular system there is a "privileged" extension of the observation operator, in the sense of Theorem [3.4, which has some special properties.

We use the same standing assumptions and notation as in Section 3 First we turn our attention to certain extensions of $C$, introduced in [39] and [45].

Definition 4.1. Let $X$ and $Y$ be Hilbert spaces, let $\mathbb{T}$ be a strongly continuous semigroup on $X$ and let $C \in \mathcal{L}\left(X_{1}, Y\right)$. The weak $L$-extension of $C$ and the weak $\Lambda$-extension of $C$ are the operators

$$
\begin{aligned}
& C_{L w} x_{0}=\text { weak } \lim _{\tau \downarrow 0} C \frac{1}{\tau} \int_{0}^{\tau} \mathbb{T}_{\sigma} x_{0} \mathrm{~d} \sigma, \\
& C_{\Lambda w} x_{0}=\text { weak } \lim _{\lambda \rightarrow+\infty} C \lambda(\lambda I-A)^{-1} x_{0},
\end{aligned}
$$

with their domains $\mathcal{D}\left(C_{L w}\right)$ and $\mathcal{D}\left(C_{\Lambda w}\right)$ consisting of those $x_{0} \in X$ for which the respective weak limits exist. Take $\lambda_{0} \in \mathbb{R}$ such that $\left[\lambda_{0}, \infty\right) \subset \rho(A)$. We define on $\mathcal{D}\left(C_{L w}\right)$ and $\mathcal{D}\left(C_{\Lambda w}\right)$ the norms

$$
\begin{aligned}
& \left\|x_{0}\right\|_{\mathcal{D}\left(C_{L w}\right)}=\left\|x_{0}\right\|_{X}+\sup _{\tau \in(0,1]}\left\|C \frac{1}{\tau} \int_{0}^{\tau} \mathbb{T}_{\sigma} x_{0} \mathrm{~d} \sigma\right\|_{Y}, \\
& \left\|x_{0}\right\|_{\mathcal{D}\left(C_{\Lambda_{w}}\right)}=\left\|x_{0}\right\|_{X}+\sup _{\lambda \in\left[\lambda_{0}, \infty\right)}\left\|C \lambda(\lambda I-A)^{-1} x_{0}\right\|_{Y} .
\end{aligned}
$$

The strong $L$-extension of $C$, denoted $C_{L}$, and the strong $\Lambda$-extension of $C$, denoted $C_{\Lambda}$, are defined in the same way, but with weak limits replaced by strong limits 1 The norms on $\mathcal{D}\left(C_{L}\right)$ and $\mathcal{D}\left(C_{\Lambda}\right)$ are the same as the norms on $\mathcal{D}\left(C_{L w}\right)$ and $\mathcal{D}\left(C_{\Lambda w}\right)$.

We remark that in Part I and 39] $C_{L}$ was called the "Lebesgue extension" of $C$, but for technical reasons we do not think that this name is suitable. We also remark that $C_{\Lambda}$ was denoted by $\widetilde{C}_{L}$ in Part I, but this notation was later changed to $C_{\Lambda}$ in 42 and this is used in several other works. Strictly speaking, $C_{L w}$ and $C_{L}$ are not needed to develop the theory in this paper: $C_{\Lambda w}$ and $C_{\Lambda}$ would be enough. However, we discuss all four extensions of $C$ in order to clarify the relation between this material and earlier work, in particular Part I.

Proposition 4.2. With the notation of Definition 4.1, $\mathcal{D}\left(C_{L_{w}}\right), \mathcal{D}\left(C_{\Lambda_{w}}\right), \mathcal{D}\left(C_{L}\right)$ and $\mathcal{D}\left(C_{\Lambda}\right)$ are Banach spaces, the corresponding extensions of $C$ are bounded linear

\footnotetext{
${ }^{1}$ The existence of these limits means that $\Psi_{\infty} x$ has the Cesàro mean of order one $C_{L} x$ or the Abel mean $C_{\Lambda} x$ at zero; cf. [7, pp. 504-505]. For this reason Staffans [30 31] uses the names Cesàro extension and Abel extension for $C_{L}$ and $C_{\Lambda}$.
} 
operators from their domains into $Y$, and any two of these extensions coincide on the intersection of their domains. Moreover,

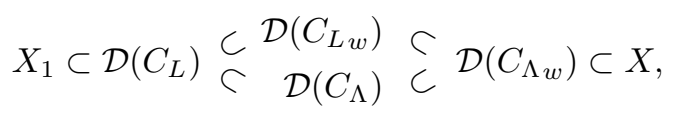

with continuous embeddings, and all the inclusions may be strict, even if $C$ is required to be admissible. If $C$ is admissible, then the space $X_{1}$ is dense in $\mathcal{D}\left(C_{L}\right)$.

Proof. The completeness of $\mathcal{D}\left(C_{L}\right)$ was proved in [39. Proposition 4.3], and the proof of completeness for the other spaces is similar, except the space $C([0,1], Y)$ appearing there has to be replaced by the space of continuous $Y$-valued functions on $(0,1]$ or on $\left[\lambda_{0}, \infty\right)$ which have a (weak) limit at 0 or $\infty$, with the sup-norm. It is obvious that $X_{1} \subset \mathcal{D}\left(C_{L}\right)$ and that $\mathcal{D}\left(C_{L}\right)$ and $\mathcal{D}\left(C_{\Lambda}\right)$ are closed subspaces of $\mathcal{D}\left(C_{L w}\right)$ and $\mathcal{D}\left(C_{\Lambda w}\right)$, respectively. The inclusion $\mathcal{D}\left(C_{L}\right) \subset \mathcal{D}\left(C_{\Lambda}\right)$ is contained in Remark I.5.7, and the corresponding weak inclusion is proved in the same way. It is easy to construct examples where the inclusions $X_{1} \subset \mathcal{D}\left(C_{L}\right)$ and $\mathcal{D}\left(C_{\Lambda w}\right) \subset X$ are strict (we leave this to the reader). The fact that the inclusions $\mathcal{D}\left(C_{L}\right) \subset \mathcal{D}\left(C_{L w}\right)$ and $\mathcal{D}\left(C_{\Lambda}\right) \subset \mathcal{D}\left(C_{\Lambda_{w}}\right)$ may be strict follows from an example with an admissible $C$ given in a sequel to this paper, which we refer to as "Part III". (The example belongs to "Part III" because it illustrates a point about duality, which is discussed there.) It is shown in Katsnelson and Weiss [11, Example 2.7] that the inclusion $\mathcal{D}\left(C_{L}\right) \subset \mathcal{D}\left(C_{\Lambda}\right)$ may be strict, again with admissible $C$. In the example constructed there, $Y=\mathbb{C}$, so that $\mathcal{D}\left(C_{L w}\right)=\mathcal{D}\left(C_{L}\right)$ and $\mathcal{D}\left(C_{\Lambda w}\right)=\mathcal{D}\left(C_{\Lambda}\right)$; hence also the inclusion $\mathcal{D}\left(C_{L w}\right) \subset \mathcal{D}\left(C_{\Lambda w}\right)$ may be strict. The fact that (with admissible $\left.C\right) X_{1}$ is dense in $\mathcal{D}\left(C_{L}\right)$ is proved in [42, Theorem 5.2].

It is still an open problem whether the inclusion $X_{1} \subset \mathcal{D}\left(C_{\Lambda}\right)$ is dense, assuming that $C$ is admissible. See Section 5 in 42 .

We denote by $\chi$ the characteristic function of $[0, \infty)$ (i.e., $\chi(t)=1$ for all $t \geq 0$ ).

Definition 4.3. For any $\mathrm{v} \in U$, the function $y_{\mathrm{v}}=\mathbb{F}_{\infty}(\chi \cdot \mathrm{v})$ is the step response of $\Sigma$ corresponding to $\mathrm{v}$. The system $\Sigma$ is called weakly regular if the following weak limit exists in $Y$, for every $\mathrm{v} \in U$ :

$$
\text { weak } \lim _{\tau \rightarrow 0} \frac{1}{\tau} \int_{0}^{\tau} y_{\mathrm{v}}(\sigma) \mathrm{d} \sigma=D \mathrm{v} .
$$

$\Sigma$ is called regular if the above limit exists in the norm topology. In either case, the operator $D \in \mathcal{L}(U ; Y)$ defined by (4.1) is called the feedthrough operator of $\Sigma$.

The fact that $D$ is bounded follows from the uniform boundedness theorem. If $Y$ is finite-dimensional, then weak regularity equals regularity, of course. In general, this is not true, as demonstrated by the same example in "Part III" that was mentioned a little earlier. The significant use of regularity is that it simplifies the computation of the operators $A, B, C$ and $D$ for closed-loop systems, see [42. Both weak and strong regularity are used in quadratic optimal control, see for example [30], 32] and [45]. We do not deal with these issues in this paper.

The following result is the weak analogue of Theorem I.2.3 and Remark I.6.2.

Theorem 4.4. Let $\Sigma=(\mathbb{T}, \Phi, \Psi, \mathbb{F})$ be a weakly regular well-posed linear system, with input space $U$, state space $X$, output space $Y$, semigroup generator $A$, control operator $B$, observation operator $C$ and feedthrough operator $D$. 
Then the output $y$ of $\Sigma$ defined in (2.14) is given by

$$
y(t)=C_{L w} x(t)+D u(t),
$$

for almost all $t \geq 0$ (in particular, $x(t) \in \mathcal{D}\left(C_{L_{w}}\right)$ for almost all $t \geq 0$ ). If $t \geq 0$ is such that both $u$ and $y$ are weakly continuous from the right at $t$, then (using those right limits) (4.2) holds at $t$ (in particular, $x(t) \in \mathcal{D}\left(C_{L w}\right)$ ).

The proof is very similar to the one for regular systems (see Section I.6), and will be omitted (in (I.4.8) we have to take the weak limits of all terms as $\tau \rightarrow 0$ ).

Theorem 4.4 implies the following formula for $\mathbb{F}_{\infty}$ for weakly regular systems (exactly as Theorem I.2.3 implies (I.2.16)):

$$
\left(\mathbb{F}_{\infty} u\right)(t)=C_{L w} \int_{0}^{t} \mathbb{T}_{t-\sigma} B u(\sigma) \mathrm{d} \sigma+D u(t),
$$

valid for every $u \in L_{\text {loc }}^{2}([0, \infty) ; U)$ and almost every $t \geq 0$ (in particular, the integral above is in $\mathcal{D}\left(C_{L w}\right)$ for almost every $\left.t \geq 0\right)$. It follows from Proposition 4.2 that in (4.2) and (4.3), $C_{L_{w}}$ may be replaced by $C_{\Lambda_{w}}$, and if $\Sigma$ is regular then (by Theorem I.2.3) also by $C_{L}$ or by $C_{\Lambda}$.

The operators $A, B, C$ and $D$ are called the generating operators of $\Sigma$, because $\Sigma$ is completely determined by them via (2.15) and 4.2.

The following is the weak version of Theorem I.4.7.

Theorem 4.5. With the notation of Theorem 4.4, assume that $\Sigma$ is weakly regular. Let $\omega_{\mathbb{T}}$ be the growth bound of $\mathbb{T}$. Then the transfer function $\mathbf{G}$ of $\Sigma$ is given by

$$
\mathbf{G}(s)=C_{L w}(s I-A)^{-1} B+D, \quad \operatorname{Re} s>\omega_{\mathbb{T}}
$$

(in particular, $(s I-A)^{-1} B U \subset \mathcal{D}\left(C_{L w}\right)$ ).

To prove this, it suffices to take weak limits in (2.18) as $\beta \rightarrow+\infty$. Note that this theorem implies that the space $Z$ defined before Theorem 3.4 is contained in $\mathcal{D}\left(C_{L w}\right)$. Hence, $\bar{C}$ from Theorem 3.4 can be chosen to be $C_{L w}$ restricted to $Z$. The formula for $\mathbf{G}$ given above is the same as (3.14) with this choice of $\bar{C}$.

The following is the weak version of Theorem I.5.8. We recall (from Section I.5) a notation for angular domains in $\mathbb{C}$ : for any $\psi \in(0, \pi)$,

$$
\mathcal{W}(\psi)=\left\{r e^{i \phi} \mid r \in(0, \infty), \phi \in(-\psi, \psi)\right\} .
$$

Theorem 4.6. Let $\Sigma=(\mathbb{T}, \Phi, \Psi, \mathbb{F})$ be a well-posed linear system, with input space $U$, state space $X$, output space $Y$, semigroup generator $A$, control operator $B$, observation operator $C$, transfer function $\mathbf{G}$, and growth bound $\omega_{\mathbb{T}}$. Then the following statements are equivalent:

(1) $\Sigma$ is weakly regular, i.e., for every $\mathrm{v} \in U$ the weak limit in (4.1) exists.

(2) For every $s \in \rho(A)$ we have that

$$
(s I-A)^{-1} B U \subset \mathcal{D}\left(C_{L w}\right)
$$

and $C_{L w}(s I-A)^{-1} B$ is an analytic $\mathcal{L}(U ; Y)$-valued function of $s$ on $\rho(A)$, uniformly bounded on any half-plane $\mathbb{C}_{\omega}$ with $\omega>\omega_{\mathbb{T}}$.

(3) There exists $s \in \rho(A)$ such that $(s I-A)^{-1} B U \subset \mathcal{D}\left(C_{L w}\right)$.

(4) There exists $s \in \rho(A)$ such that $(s I-A)^{-1} B U \subset \mathcal{D}\left(C_{\Lambda w}\right)$.

(5) Any state trajectory of $\Sigma$ is almost always in $\mathcal{D}\left(C_{L w}\right)$.

(6) Any state trajectory of $\Sigma$ is almost always in $\mathcal{D}\left(C_{\Lambda w}\right)$. 
(7) For every $\mathrm{v} \in U$ and every $\psi \in(0, \pi / 2), \mathbf{G}(s) \mathrm{v}$ has a weak limit as $|s| \rightarrow \infty$ and $s \in \mathcal{W}(\psi)$.

(8) For every $\mathrm{v} \in U, \mathbf{G}(\lambda) \mathrm{v}$ has a weak limit as $\lambda \rightarrow+\infty$ in $\mathbb{R}$.

Moreover, if the limits mentioned in statements (1), (7) and (8) above exist, then they are equal to $D \mathrm{v}$, where $D$ is the feedthrough operator of $\Sigma$.

The proof is similar to that of Theorem I.5.8, and we leave the details to the reader. Observe that $(s I-A)^{-1} B U$ in conditions (2)-(4) could be replaced by the space $Z$ defined in Section 3, both in Theorem I.5.8 and in Theorem 4.6.

\section{System BeHAVIOR FOR NEGATIVE TIME}

Until now we have considered the time to be positive. It is sometimes important to think of a well-posed linear system $\Sigma$ functioning on the time interval $(-\infty, 0]$ or $(-\infty, \infty)$. To treat these cases, we introduce some further notation and we extend $\Phi_{t}$ and $\mathbb{F}_{\infty}$ so that they depend also on the values of the input for negative times. Some results related to those in this section were given in [30, [31] and [45].

Notation 5.1. Let $W$ be a Hilbert space. The operator $\mathcal{S}_{\tau}$ (with $\tau \in \mathbb{R}$ ) is the (bilateral) right shift by $\tau$ on $L_{\text {loc }}^{2}((-\infty, \infty) ; W)$, so that $\mathcal{S}_{-\tau}$ denotes the (bilateral) left shift by $\tau$ on the same space. Recall the projections $\mathbf{P}_{-}, \mathbf{P}_{+}$and the spaces $L_{\omega}^{2}(J ; W)$ introduced in Section 2 . The space $L_{\omega, \text { loc }}^{2}((-\infty, \infty) ; W)$ consists of all the functions $u \in L_{\text {loc }}^{2}((-\infty, \infty) ; W)$ for which $\mathbf{P}_{-} u \in L_{\omega}^{2}((-\infty, 0] ; W)$. We regard $L_{\omega, \text { loc }}^{2}((-\infty, \infty) ; W)$ as a Fréchet space, with the metric generated by the seminorms

$$
\|u\|_{n}=\left(\int_{-\infty}^{n} e^{-2 \omega t}\|u(t)\|^{2} \mathrm{~d} t\right)^{1 / 2}, \quad n \in \mathbb{N} .
$$

The unilateral right shift $\mathbf{S}_{\tau}$ (with $\tau \geq 0$ ) was originally defined on $L_{\text {loc }}^{2}([0, \infty) ; W)$, but we extend it to $L_{\text {loc }}^{2}((-\infty, \infty) ; W)$ by $\mathbf{S}_{\tau}=\mathcal{S}_{\tau} \mathbf{P}_{+}$. Note that $\mathbf{S}_{\tau}=\mathbf{P}_{[\tau, \infty)} \mathcal{S}_{\tau}$.

Throughout this section, we assume that $\Sigma=(\mathbb{T}, \Phi, \Psi, \mathbb{F})$ is a well-posed linear system with input space $U$, state space $X$, output space $Y$, transfer function $\mathbf{G}$ and growth bounds $\omega_{\mathbb{T}}$ and $\gamma_{\mathbb{F}}$, and we use all the notation introduced in Section 2 Note that $\Phi_{t}$ was originally defined on $L^{2}([0, \infty) ; U)$, but (2.4) shows that $\Phi_{t}$ has an obvious extension to $L_{\text {loc }}^{2}((-\infty, \infty) ; U)$, still given by $(2.6)$.

Proposition 5.2. For all $u \in L_{\omega, \mathrm{loc}}^{2}((-\infty, \infty) ; U)$ with $\omega>\omega_{\mathbb{T}}$ and for all $t \in \mathbb{R}$, the following limit exists in $X$ :

$$
\widetilde{\Phi}_{t} u=\lim _{\tau \rightarrow \infty} \Phi_{\tau+t} \mathcal{S}_{\tau} u .
$$

We have

$$
\widetilde{\Phi}_{t} u=\int_{-\infty}^{t} \mathbb{T}_{t-\sigma} B u(\sigma) \mathrm{d} \sigma
$$

and there exists a $k_{\omega} \geq 0$ (independent of $t$ and $u$ ) such that

$$
\left\|\widetilde{\Phi}_{t} u\right\| \leq k_{\omega} e^{\omega t}\left\|\mathbf{P}_{(-\infty, t]} u\right\|_{L_{\omega}^{2}}
$$

Proof. By (2.6), for all $t \in \mathbb{R}$ and all $\tau \geq-t$,

$$
\Phi_{\tau+t} \mathcal{S}_{\tau} u=\int_{0}^{\tau+t} \mathbb{T}_{\tau+t-\sigma} B u(\sigma-\tau) \mathrm{d} \sigma=\int_{-\tau}^{t} \mathbb{T}_{t-\sigma} B u(\sigma) \mathrm{d} \sigma .
$$


Thus, to show that the limit in (5.1) exists, we have to show that (in $X$ )

$$
\left\|\Phi_{T+t} \mathcal{S}_{T} u-\Phi_{\tau+t} \mathcal{S}_{\tau} u\right\|=\left\|\int_{-T}^{-\tau} \mathbb{T}_{t-\sigma} B u(\sigma) \mathrm{d} \sigma\right\|
$$

tends to zero as $\tau, T \rightarrow \infty$. Without loss of generality, assume that $T \geq \tau$.

Denote $v(t)=e^{-\omega t} u(t)$ and $\widetilde{\mathbb{T}}_{t}=e^{-\omega t} \mathbb{T}_{t}$, so that $\mathbf{P}_{(-\infty, t]} v \in L^{2}((-\infty, t] ; U)$ and the semigroup $\widetilde{\mathbb{T}}$ is exponentially stable $\left(\omega_{\widetilde{\mathbb{T}}}<0\right)$. Then

$$
\begin{aligned}
\left\|\Phi_{T+t} \mathcal{S}_{T} u-\Phi_{\tau+t} \mathcal{S}_{\tau} u\right\| & =e^{\omega t}\left\|\int_{-T}^{-\tau} \widetilde{\mathbb{T}}_{t-\sigma} B v(\sigma) \mathrm{d} \sigma\right\| \\
& \leq e^{\omega t}\left\|\widetilde{\mathbb{T}}_{\tau+t}\right\| \cdot\left\|\int_{-T}^{-\tau} \widetilde{\mathbb{T}}_{-\tau-\sigma} B v(\sigma) \mathrm{d} \sigma\right\| .
\end{aligned}
$$

Denote $q=\mathcal{S}_{T} v$, so that $\left\|\mathbf{P}_{(-\infty, T+t]} q\right\|=\left\|\mathbf{P}_{(-\infty, t]} v\right\|$. We have

$$
\left\|\Phi_{T+t} \mathcal{S}_{T} u-\Phi_{\tau+t} \mathcal{S}_{\tau} u\right\| \leq e^{\omega t}\left\|\widetilde{\mathbb{T}}_{\tau+t}\right\| \cdot\left\|\int_{0}^{T-\tau} \widetilde{\mathbb{T}}_{T-\tau-\nu} B q(\nu) \mathrm{d} \nu\right\| .
$$

Since $B$ is admissible for $\widetilde{\mathbb{T}}$ and $\widetilde{\mathbb{T}}$ is exponentially stable, the third factor above is bounded by $k_{\omega}\left\|\mathbf{P}_{T-\tau} q\right\|$, with $k_{\omega}$ independent of $T, \tau$ and $q$, see [38, Remark 2.6]. Since

$$
\left\|\mathbf{P}_{T-\tau} q\right\| \leq\left\|\mathbf{P}_{T+t} q\right\| \leq\left\|\mathbf{P}_{(-\infty, T+t]} q\right\|=\left\|\mathbf{P}_{(-\infty, t]} v\right\|
$$

we get that

$$
\left\|\Phi_{T+t} \mathcal{S}_{T} u-\Phi_{\tau+t} \mathcal{S}_{\tau} u\right\| \leq k_{\omega} e^{\omega t}\left\|\widetilde{\mathbb{T}}_{\tau+t}\right\| \cdot\left\|\mathbf{P}_{(-\infty, t]} v\right\| .
$$

The last expression tends to zero as $\tau \rightarrow \infty$, so that the limit in (5.1) exists.

Combining the definition (5.1) of $\widetilde{\Phi}_{t}$ with (5.4), we obtain (5.2). The integral in (5.2) exists in $X_{-1}$ because what we integrate is in $L^{1}\left((-\infty, t] ; X_{-1}\right)$, as is easy to see (but now we know from (5.1) that the integral is in $X$ ). Finally, (5.3) follows from (5.6) by taking $\tau=-t$ and using (5.1) (with $T$ in place of $\tau$ ).

We call the operators $\widetilde{\Phi}_{t}$ from (5.1) the extended input maps of $\Sigma$. Using (2.1) to express $\Phi_{\tau+t}$ in (5.1), we obtain that for all $t \geq 0$,

$$
\widetilde{\Phi}_{t}=\mathbb{T}_{t} \widetilde{\Phi}_{0}+\Phi_{t}
$$

By replacing $\tau$ by $T, t$ by $\tau+t$ and $u$ by $\mathcal{S}_{\tau} u$ in (5.1), we find that for all $t, \tau \in \mathbb{R}$, $\widetilde{\Phi}_{\tau+t} \mathcal{S}_{\tau}=\widetilde{\Phi}_{t}$. Multiplying this by $\mathcal{S}_{-\tau}$ to the right and using (5.7), we get the following extension of (2.1): for all $\tau \in \mathbb{R}$ and all $t \geq 0$,

$$
\widetilde{\Phi}_{\tau+t}=\mathbb{T}_{t} \widetilde{\Phi}_{\tau}+\Phi_{t} \mathcal{S}_{-\tau} \text {. }
$$

Recall that for each $\omega>\gamma_{\mathbb{F}}, \mathbb{F}_{\infty}$ is a bounded operator from $L_{\omega}^{2}([0, \infty) ; U)$ to $L_{\omega}^{2}([0, \infty) ; Y)$, and we denote by $\left\|\mathbb{F}_{\infty}\right\|_{\omega}$ the corresponding operator norm.

Proposition 5.3. For all $u \in L_{\omega, \mathrm{loc}}^{2}((-\infty, \infty) ; U)$ with $\omega>\gamma_{\mathbb{F}}$, the following limit exists in $L_{\omega, \mathrm{loc}}^{2}((-\infty, \infty) ; Y)$ :

$$
\mathcal{F} u=\lim _{\tau \rightarrow \infty} \mathcal{S}_{-\tau} \mathbb{F}_{\infty} \mathcal{S}_{\tau} u
$$

The operator $\mathcal{F}$ defined in this way is a bilaterally shift-invarant and causal extension of $\mathbb{F}_{\infty}$, which means that

$$
\mathcal{F} \mathbf{P}_{+}=\mathbb{F}_{\infty}, \quad \mathcal{F} \mathcal{S}_{t}=\mathcal{S}_{t} \mathcal{F}, \quad \mathbf{P}_{(-\infty, t]} \mathcal{F} \mathbf{P}_{[t, \infty)}=0
$$


for all $t \in \mathbb{R}$. For each $\omega>\gamma_{\mathbb{F}}, \mathcal{F}$ maps $L_{\omega}^{2}((-\infty, \infty) ; U)$ into $L_{\omega}^{2}((-\infty, \infty) ; Y)$, and we denote by $\|\mathcal{F}\|_{\omega}$ the corresponding operator norm. We have

$$
\|\mathcal{F}\|_{\omega}=\left\|\mathbb{F}_{\infty}\right\|_{\omega}=\sup _{s \in \mathbb{C}_{\omega}}\|\mathbf{G}(s)\|
$$

Proof. Let $u \in L_{\omega, \text { loc }}^{2}((-\infty, \infty) ; U)$. To prove that the limit on the right-hand side of (5.9) exists, it suffices to show that the difference

$$
\mathcal{S}_{-T} \mathbb{F}_{\infty} \mathcal{S}_{T} u-\mathcal{S}_{-\tau} \mathbb{F}_{\infty} \mathcal{S}_{\tau} u=\mathcal{S}_{-T}\left(\mathbb{F}_{\infty}-\mathcal{S}_{T-\tau} \mathbb{F}_{\infty} \mathcal{S}_{\tau-T}\right) \mathcal{S}_{T} u
$$

tends to zero in $L_{\omega}^{2}(\mathbb{R} ; Y)$ as $\tau, T \rightarrow \infty$ with $T \geq \tau$. Multiplying $(2.12)$ by $\mathbf{S}_{\tau}^{*}$ to the right, and then replacing $\tau$ by $T-\tau$, we get the second equality in

$$
\mathcal{S}_{T-\tau} \mathbb{F}_{\infty} \mathcal{S}_{\tau-T}=\mathbf{S}_{T-\tau} \mathbb{F}_{\infty} \mathbf{S}_{T-\tau}^{*}=\mathbb{F}_{\infty} \mathbf{P}_{[T-\tau, \infty)},
$$

so that, using $I-\mathbf{P}_{[T-\tau, \infty)}=\mathbf{P}_{[0, T-\tau]}$, we obtain

$$
\mathcal{S}_{-T} \mathbb{F}_{\infty} \mathcal{S}_{T} u-\mathcal{S}_{-\tau} \mathbb{F}_{\infty} \mathcal{S}_{\tau} u=\mathcal{S}_{-T} \mathbb{F}_{\infty} \mathbf{P}_{[0, T-\tau]} \mathcal{S}_{T} u=\mathcal{S}_{-T} \mathbb{F}_{\infty} \mathcal{S}_{T} \mathbf{P}_{[-T,-\tau]} u
$$

This implies

$$
\left\|\mathcal{S}_{-T} \mathbb{F}_{\infty} \mathcal{S}_{T} u-\mathcal{S}_{-\tau} \mathbb{F}_{\infty} \mathcal{S}_{\tau} u\right\|_{L_{\omega}^{2}} \leq\left\|\mathcal{S}_{-T}\right\|_{\omega} \cdot\left\|\mathbb{F}_{\infty}\right\|_{\omega} \cdot\left\|\mathcal{S}_{T}\right\|_{\omega} \cdot\left\|\mathbf{P}_{[-T,-\tau]} u\right\|_{L_{\omega}^{2}},
$$

where $\left\|\mathcal{S}_{-T}\right\|_{\omega}$ is the norm of $\mathcal{S}_{-T}$ on the space $L_{\omega}^{2}((-\infty, \infty) ; Y)$, which is easily seen to be $e^{\omega T}$, and similarly $\left\|\mathcal{S}_{T}\right\|_{\omega}=e^{-\omega T}$. Thus

$$
\left\|\mathcal{S}_{-T} \mathbb{F}_{\infty} \mathcal{S}_{T} u-\mathcal{S}_{-\tau} \mathbb{F}_{\infty} \mathcal{S}_{\tau} u\right\|_{L_{\omega}^{2}} \leq\left\|\mathbb{F}_{\infty}\right\|_{\omega} \cdot\left\|\mathbf{P}_{[-T,-\tau]} u\right\|_{L_{\omega}^{2}}
$$

and the right-hand side tends to zero as $\tau, T \rightarrow \infty$ with $T \geq \tau$, showing that the limit in (5.9) exists. The same argument shows that if $u \in L_{\omega}^{2}(\mathbb{R} ; U)$, then the limit on the right-hand side of (5.9) exists in $L_{\omega}^{2}(\mathbb{R} ; Y)$, so $\mathcal{F}$ maps $L_{\omega}^{2}(\mathbb{R} ; U)$ into $L_{\omega}^{2}(\mathbb{R} ; Y)$. The identity $\mathcal{F} \mathbf{P}_{+}=\mathbb{F}_{\infty}$ follows from (2.12) and (5.9), using that $\mathbf{S}_{\tau}=\mathcal{S}_{\tau} \mathbf{P}_{+}$. To get the shift-invariance (the second part of (5.10)), it suffices to replace $u$ by $\mathcal{S}_{t} u$ in (5.9). The causality (the third part of (5.10) ) follows from the shift-invariance of $\mathcal{F}$ and the fact that $\mathbf{P}_{-} \mathcal{F} \mathbf{P}_{+}=\mathbf{P}_{-} \mathbb{F}_{\infty}=0$. To prove that $\|\mathcal{F}\|_{\omega} \leq\left\|\mathbb{F}_{\infty}\right\|_{\omega}$, we use (15.9) with the limit taken in $L_{\omega}^{2}(\mathbb{R} ; Y)$ : we have, for all $\tau \in \mathbb{R}$,

$$
\left\|\mathcal{S}_{-\tau} \mathbb{F}_{\infty} \mathcal{S}_{\tau} u\right\|_{L_{\omega}^{2}} \leq\left\|\mathcal{S}_{-\tau}\right\|_{\omega} \cdot\left\|\mathbb{F}_{\infty}\right\|_{\omega} \cdot\left\|\mathcal{S}_{\tau}\right\|_{\omega} \cdot\|u\|_{L_{\omega}^{2}}=\left\|\mathbb{F}_{\infty}\right\|_{\omega} \cdot\|u\|_{L_{\omega}^{2}}
$$

hence $\|\mathcal{F} u\|_{L_{\omega}^{2}} \leq\left\|\mathbb{F}_{\infty}\right\|_{\omega} \cdot\|u\|_{L_{\omega}^{2}}$. The inequality $\|\mathcal{F}\|_{\omega} \geq\left\|\mathbb{F}_{\infty}\right\|_{\omega}$ is trivial, since $\mathbb{F}_{\infty}=\mathcal{F} \mathbf{P}_{+}$and $\left\|\mathbf{P}_{+}\right\|_{\omega}=1$. Finally, the fact that $\left\|\mathbb{F}_{\infty}\right\|_{\omega}=\sup _{s \in \mathbb{C}_{\omega}}\|\mathbf{G}(s)\|$ was proved in Theorem I.3.6, so that (5.11) holds.

By replacing $\tau$ in (2.11) by $\tau+T$, multiplying by $\mathcal{S}_{T} u$ to the right, by $\mathcal{S}_{-T}$ to the left, and letting $T \rightarrow \infty$ we get the following extension of (2.11): for all $\tau \in \mathbb{R}$,

$$
\mathcal{F}=\mathbf{P}_{(-\infty, \tau]} \mathcal{F}+\mathcal{S}_{\tau} \Psi_{\infty} \widetilde{\Phi}_{\tau}+\mathcal{S}_{\tau} \mathbb{F}_{\infty} \mathcal{S}_{-\tau}
$$

Remark 5.4. In the formulation of Staffans [30, 31, a well-posed linear system is defined in terms the semigroup $\mathbb{T}_{t}$ (denoted by $\mathcal{A}(t)$ ) and the extended operators $\widetilde{\Phi}_{0}$ $($ denoted by $\mathcal{B}), \Psi_{\infty}($ denoted by $\mathcal{C})$, and $\mathcal{F}$ (denoted by $\left.\mathcal{D}\right)$. The original operator families of input maps $\Phi_{\tau}$, output maps $\Psi_{\tau}$, and and input-output maps $\mathbb{F}_{\tau}$ can be recovered from $\widetilde{\Phi}_{0}, \Psi_{\infty}$, and $\mathcal{F}$ by means of

$$
\Phi_{\tau}=\widetilde{\Phi}_{0} \mathcal{S}_{-\tau} \mathbf{P}_{+}, \quad \Psi_{\tau}=\mathbf{P}_{\tau} \Psi_{\infty}, \quad \mathbb{F}_{\tau}=\mathbf{P}_{\tau} \mathcal{F} \mathbf{P}_{\tau}
$$


Moreover, Staffans writes the algebraic conditions (2.1)-(2.3) as (in our notation)

$$
\begin{aligned}
\mathbb{T}_{t} \widetilde{\Phi}_{0} & =\widetilde{\Phi}_{0} \mathcal{S}_{-t} \mathbf{P}_{-}, \quad t \geq 0, \\
\Psi_{\infty} \mathbb{T}_{t} & =\mathbf{S}_{t}^{*} \Psi_{\infty}, \quad t \geq 0, \\
\mathbf{P}_{-} \mathcal{F} \mathbf{P}_{+} & =0, \quad \mathbf{P}_{+} \mathcal{F} \mathbf{P}_{-}=\Psi_{\infty} \widetilde{\Phi}_{0}, \quad \mathcal{S}_{t} \mathcal{F}=\mathcal{F} \mathcal{S}_{t}, \quad t \in \mathbb{R} .
\end{aligned}
$$

Definition 5.5. For each $u \in L_{\omega, \text { loc }}^{2}((-\infty, \infty) ; U)$ with $\omega>\omega_{\mathbb{T}}$ we define the corresponding state trajectory vanishing at $-\infty, x:(-\infty, \infty) \rightarrow X$, and the corresponding bilateral output function $y \in L_{\omega, \mathrm{loc}}^{2}((-\infty, \infty) ; Y)$ by

$$
x(t)=\widetilde{\Phi}_{t} u, \quad y=\mathcal{F} u .
$$

These concepts of state trajectory and output function are identical to those used by Staffans [30, Definition 9], 31, Definition 2.2] in "the time-invariant setting."

The following theorem represents the "bilateral" version of Proposition 2.6 and Theorems 3.1, 3.2 and 4.4. We use the notation $C \& D,[C \& D]_{\Lambda}$ and $S_{\Sigma}(s)$ introduced in Section 3, as well as $C_{L w}$ and $C_{\Lambda w}$ from Section 4.

Theorem 5.6. Denote the generator of $\mathbb{T}$ by $A$, the control operator of $\Sigma$ by $B$, the observation operator of $\Sigma$ by $C$ and the combined observation/feedthrough operator of $\Sigma$ by $C \& D$. Let $\omega>\omega_{\mathbb{T}}$ and $u \in L_{\omega, \text { loc }}^{2}((-\infty, \infty) ; U)$, and define the state trajectory $x$ and the output function $y$ as in (5.13).

(i) The function $x$ is the unique strong solution in $X_{-1}$ of

$$
\begin{aligned}
& \dot{x}(t)=A x(t)+B u(t), \quad t \in \mathbb{R}, \\
& \lim _{t \rightarrow-\infty} e^{-\omega t} x(t)=0,
\end{aligned}
$$

i.e., $x$ is continuous with values in $X, x \in H_{\mathrm{loc}}^{1}\left((-\infty, \infty) ; X_{-1}\right)$, and its derivative $\dot{x}$ satisfies (5.14) for almost every $t \in \mathbb{R}$. Moreover, for almost every $t \in \mathbb{R}$ we have that $\left[\begin{array}{l}x(t) \\ u(t)\end{array}\right] \in \mathcal{D}\left([C \& D]_{\Lambda}\right)$ and the output $y(t)$ is given by (3.7).

(ii) If $u \in H_{\omega}^{1}((-\infty, \infty) ; U)$, then

$x \in C^{1}((-\infty, \infty) ; X), \quad\left[\begin{array}{l}x \\ u\end{array}\right] \in C((-\infty, \infty) ; V), \quad y \in H_{\omega}^{1}((-\infty, \infty) ; Y)$,

and for every $t \in \mathbb{R}$, the formula (3.3) holds.

(iii) If $\Sigma$ is weakly regular, and if we denote the feedthrough operator of $\Sigma$ by $D$, then for almost every $t \in \mathbb{R}$ we have that $x(t) \in \mathcal{D}\left(C_{L w}\right)$ and the output $y(t)$ is given by the formula (4.2). If $t \in \mathbb{R}$ is such that both $u$ and $y$ are weakly continuous from the right at $t$, then (using those right limits) (4.2) holds at $t$ (in particular, $x(t) \in \mathcal{D}\left(C_{L w}\right)$ ).

We remark that the meaning of a strong solution of $\dot{x}(t)=A x(t)+B u(t)$ in $X_{-1}$ on an interval $J$ is as in Proposition 2.6, where we had $J=[0, \infty)$.

Proof. (i) Fix $\tau \in \mathbb{R}$. By (5.8), we can interpret the function $q(t)=x(\tau+t)=$ $\widetilde{\Phi}_{\tau+t} u$, defined for $t \geq 0$, as the state trajectory of $\Sigma$ (in the sense of Definition [2.4) with initial state $x_{0}=\widetilde{\Phi}_{\tau} u$ and input function $v=\mathbf{P}_{+} \mathcal{S}_{-\tau} u$. By (5.12), the function $w$ defined by $w=\mathbf{P}_{+} \mathcal{S}_{-\tau} y$ is the corresponding output function. Indeed, by (5.12),

$$
w=\mathbf{P}_{+} \mathcal{S}_{-\tau} \mathcal{F} u=\Psi_{\infty} \widetilde{\Phi}_{\tau} u+\mathbb{F}_{\infty} \mathcal{S}_{-\tau} u=\Psi_{\infty} x_{0}+\mathbb{F}_{\infty} v
$$


By Proposition [2.6, $q$ is a strong solution in $X_{-1}$ of $\dot{q}(t)=A q(t)+B v(t)$ on $[0, \infty)$, whence $x$ is a strong solution in $X_{-1}$ of $\dot{x}(t)=A x(t)+B u(t)$ on $[\tau, \infty)$. By Theorem $3.2\left[\begin{array}{l}q(t) \\ v(t)\end{array}\right] \in \mathcal{D}\left([C \& D]_{\Lambda}\right)$ and $w(t)=[C \& D]_{\Lambda}\left[\begin{array}{l}q(t) \\ v(t)\end{array}\right]$ hold for almost every $t \geq 0$. As $\tau$ was arbitrary, $x$ is a strong solution in $X_{-1}$ of $\dot{x}(t)=A x(t)+B u(t)$ on $(-\infty, \infty)$, and (3.7) holds for almost every $t \geq 0$.

To prove the uniqueness of this strong solution with the extra initial condition at $-\infty$, suppose that we have two such solutions $x_{1}$ and $x_{2}$. Then their difference $z=x_{2}-x_{1}$ is a strong solution of $\dot{z}(t)=A z(t)$ on $(-\infty, \infty)$, hence $z(t)=\mathbb{T}_{t-\tau} z(\tau)$ for all $t, \tau \in \mathbb{R}$ with $t \geq \tau$. The initial condition in (5.14) implies that $\lim _{\tau \rightarrow-\infty} \mathbb{T}_{t-\tau} z(\tau)=0$, so that $z(t)=0$ for all $t \in \mathbb{R}$, proving uniqueness.

(ii) In this case $u \in C((-\infty, \infty) ; U)$, so $A x+B u \in C\left((-\infty, \infty) ; X_{-1}\right)$. Since $x \in H_{\mathrm{loc}}^{1}\left((-\infty, \infty) ; X_{-1}\right)$ and $\dot{x}(t)=A x(t)+B u(t)$ in $X_{-1}$ for almost every $t \in \mathbb{R}$, we find that $x \in C^{1}\left((-\infty, \infty) ; X_{-1}\right)$ and that $\dot{x}(t)=A x(t)+B u(t)$ for all $t \in \mathbb{R}$. By the definition of $x$ and the comment after (5.7), for all $\tau, t \in \mathbb{R}$,

$$
\frac{x(\tau+t)-x(\tau)}{t}=\frac{\widetilde{\Phi}_{\tau+t} u-\widetilde{\Phi}_{\tau} u}{t}=\widetilde{\Phi}_{\tau} \frac{\mathcal{S}_{-t} u-u}{t}
$$

As $t \rightarrow 0$, we have $\left(\mathcal{S}_{-t} u-u\right) / t \rightarrow \dot{u}$ in $L_{\omega}^{2}((-\infty, \infty) ; U)$, so by the continuity of $\widetilde{\Phi}_{\tau}$, $x$ is differentiable at $\tau$ in the space $X$ (and $\left.\dot{x}(\tau)=\widetilde{\Phi}_{\tau} \dot{u}\right)$. Thus $A x(\tau)+B u(\tau)=$ $\dot{x}(\tau) \in X$. This means that $\left[\begin{array}{l}x(\tau) \\ u(\tau)\end{array}\right] \in V$ for all $\tau \in \mathbb{R}$. A similar argument with $x(\tau)$ replaced by $y$ and $\widetilde{\Phi}_{\tau}$ replaced by $\mathcal{F}$ shows that $y \in H_{\omega}^{1}((-\infty, \infty) ; Y$ ) (and that $\dot{y}=\mathcal{F} \dot{u})$. We can now repeat the argument in the proof of (i), with Proposition 2.6 and Theorem 3.2 replaced by Theorem 3.1 (i), to show that $x \in C^{1}((-\infty, \infty) ; X)$, $\left[\begin{array}{l}x \\ u\end{array}\right] \in C((-\infty, \infty) ; V)$, and (3.3) holds for all $t \in \mathbb{R}$.

(iii) The proof of this point is similar to the proof of (i), but now we replace Theorem 3.2 by Theorem 4.4 .

\section{The CONNECTION WITH SCATtERING THEORY}

Starting from an arbitrary well-posed linear system $\Sigma$, it is possible to define a strongly continuous semigroup which resembles those encountered in the scattering theory of Lax and Phillips [12, 13, and which contains all the information about $\Sigma$.

Notation 6.1. As in the previous section, we assume that $\Sigma=(\mathbb{T}, \Phi, \Psi, \mathbb{F})$ is a wellposed linear system with input space $U$, state space $X$, output space $Y$, transfer function $\mathbf{G}$ and the two growth bounds $\omega_{\mathbb{T}}$ and $\gamma_{\mathbb{F}}$. We continue to use the notation $\mathbf{P}_{-}, \mathbf{P}_{+}, \mathbf{S}_{t}, \mathbf{S}_{t}^{*}, \mathbb{C}_{\omega}, L_{\omega}^{2}, H_{\omega}^{1}, \Psi_{\infty}$ and $\mathbb{F}_{\infty}$ introduced in Section 2 , and $\mathcal{S}_{t}, \widetilde{\Phi}_{t}$ and $\mathcal{F}$ from Section 5 . We denote the generator of $\mathbb{T}$ by $A$, the control operator of $\Sigma$ by $B$, its observation operator by $C$, its combined observation/feedthrough operator by $C \& D$, and its system operator by $S_{\Sigma}(s)$. $C \& D$ and $S_{\Sigma}(s)$ were introduced in Section 3. The domain of $C \& D$ and of $S_{\Sigma}(s)$ is the space $V$ from (3.1).

Proposition 6.2. Let $\omega \in \mathbb{R}, \mathcal{Y}=L_{\omega}^{2}((-\infty, 0] ; Y)$ and $\mathcal{U}=L_{\omega}^{2}([0, \infty) ; U)$. For all $t \geq 0$, on $\mathcal{Y} \times X \times \mathcal{U}$ we define the operator $\mathfrak{T}_{t}$ by

$$
\boldsymbol{T}_{t}=\left[\begin{array}{ccc}
\mathcal{S}_{-t} & 0 & 0 \\
0 & I & 0 \\
0 & 0 & \mathbf{S}_{t}^{*}
\end{array}\right]\left[\begin{array}{ccc}
I & \Psi_{t} & \mathbb{F}_{t} \\
0 & \mathbb{T}_{t} & \Phi_{t} \\
0 & 0 & I
\end{array}\right]
$$


(this looks like (1.7), but we are now in a more general context). Then $\boldsymbol{T}=\left(\boldsymbol{T}_{t}\right)_{t \geq 0}$ is a strongly continuous semigroup. Take $y_{0} \in \mathcal{Y}, x_{0} \in X$ and $u_{0} \in \mathcal{U}$. We denote by $x$ the state trajectory $x(t)=\mathbb{T}_{t} x_{0}+\Phi_{t} u_{0}$ and by $y$ the "bilateral" output function, equal to $y_{0}$ for $t<0$, and equal to $\Psi_{\infty} x_{0}+\mathbb{F}_{\infty} u_{0}$ for $t \geq 0$. Then for all $t \geq 0$,

$$
\left[\begin{array}{c}
\mathbf{P}_{(-\infty, t]} y \\
x(t) \\
\mathbf{P}_{[t, \infty)} u_{0}
\end{array}\right]=\left[\begin{array}{ccc}
\mathcal{S}_{t} & 0 & 0 \\
0 & I & 0 \\
0 & 0 & \mathbf{S}_{t}
\end{array}\right] \boldsymbol{T}_{t}\left[\begin{array}{c}
y_{0} \\
x_{0} \\
u_{0}
\end{array}\right]
$$

Formula (6.1) shows that at any time $t \geq 0$, the first component of $\mathfrak{T}_{t}\left[\begin{array}{c}y_{0} \\ x_{0} \\ u_{0}\end{array}\right]$ represents the past output, the second component represents the present state and the third component represents the future input.

Proof. The semigroup property $\boldsymbol{T}_{\tau+t}=\boldsymbol{T}_{t} \boldsymbol{T}_{\tau}$ follows (via elementary algebra) from the semigroup property of $\mathbb{T}, 2.1-2.3$ and the fact that the left shifts $\mathcal{S}_{-t}$ and $\mathbf{S}_{t}^{*}$ are semigroups on $\mathcal{Y}$ and $\mathcal{U}$, respectively. In a similar way, the initial condition $\mathfrak{T}_{0}=I$ follows from the initial conditions $\mathbb{T}_{0}=I, \Phi_{0}=0, \Psi_{0}=0, \mathbb{F}_{0}=0, \mathbf{S}_{0}^{*}=I$, $\mathcal{S}_{0}=I$. The formula (6.1) is a direct consequence of Definition 2.4

To prove the strong continuity, we split $\left[\begin{array}{l}y_{0} \\ x_{0} \\ u_{0}\end{array}\right] \in \mathcal{Y} \times X \times \mathcal{U}$ into $\left[\begin{array}{c}y_{0} \\ 0 \\ 0\end{array}\right]+\left[\begin{array}{c}0 \\ x_{0} \\ u_{0}\end{array}\right]$. The continuity of $\boldsymbol{T}_{t}$ applied to the first vector follows from the strong continuity of $\mathcal{S}_{-t}$. The continuity of $\boldsymbol{T}_{t}$ applied to the second vector follows from the strong continuity of $\mathbf{S}_{t}^{*}$ and $\mathcal{S}_{-t}$ and from (6.1) (using the continuity of state trajectories).

In the case where $\omega=0$ and $\mathfrak{T}$ is contractive (or unitary), $\mathfrak{T}$ is isomorphic to a semigroup of the type studied by Lax and Phillips (the unitary case is treated in 12 and the contractive case in [13]; an extension to the general case is in Helton [6]). For this reason, we call $\mathfrak{T}$ the Lax-Phillips semigroup of index $\omega$ induced by $\Sigma$.

The generator $\mathfrak{A}$ of the Lax-Phillips semigroup and the spectrum of $\mathfrak{A}$ can be characterized as follows:

Theorem 6.3. Let $\omega \in \mathbb{R}$, and let $\boldsymbol{T}$ be the Lax-Phillips semigroup of index $\omega$ induced by $\Sigma$. We denote the generator of $\boldsymbol{T}$ by $\mathfrak{A}$.

(i) The domain of $\mathfrak{A}, \mathcal{D}(\mathfrak{A})$, consists of all the vectors $\left[\begin{array}{l}y_{0} \\ x_{0} \\ u_{0}\end{array}\right] \in H_{\omega}^{1}((-\infty, 0] ; Y) \times$ $X \times H_{\omega}^{1}([0, \infty) ; U)$ which satisfy $\left[\begin{array}{c}x_{0} \\ u_{0}(0)\end{array}\right] \in V$ and $y_{0}(0)=C \& D\left[\begin{array}{c}x_{0} \\ u_{0}(0)\end{array}\right]$, and $\mathfrak{A}$ is given on $\mathcal{D}(\boldsymbol{\mathfrak { A }})$ by

$$
\mathfrak{A}\left[\begin{array}{l}
y_{0} \\
x_{0} \\
u_{0}
\end{array}\right]=\left[\begin{array}{c}
y_{0}^{\prime} \\
A x_{0}+B u_{0}(0) \\
u_{0}^{\prime}
\end{array}\right]
$$

(ii) The following two conditions are equivalent (for any $\lambda \in \mathbb{C}$ ):

(a) $\left[\begin{array}{l}y_{0} \\ x_{0} \\ u_{0}\end{array}\right] \in \mathcal{D}(\mathfrak{A})$ and $\left[\begin{array}{l}y \\ x \\ u\end{array}\right]=(\mathfrak{A}-\lambda I)\left[\begin{array}{l}y_{0} \\ x_{0} \\ u_{0}\end{array}\right]$,

(b) $y_{0} \in H_{\omega}^{1}((-\infty, 0] ; Y), x_{0} \in X, u_{0} \in H_{\omega}^{1}([0, \infty) ; U),\left[\begin{array}{c}x_{0} \\ u_{0}(0)\end{array}\right] \in V$ and

$$
\left[\begin{array}{c}
x \\
y_{0}(0)
\end{array}\right]=S_{\Sigma}(\lambda)\left[\begin{array}{c}
x_{0} \\
u_{0}(0)
\end{array}\right], \quad\left[\begin{array}{l}
y \\
u
\end{array}\right]=\left[\begin{array}{l}
y_{0}^{\prime} \\
u_{0}^{\prime}
\end{array}\right]-\lambda\left[\begin{array}{l}
y_{0} \\
u_{0}
\end{array}\right] .
$$

(iii) The spectrum $\sigma(\mathfrak{A})$ contains the vertical line where $\operatorname{Re} \lambda=\omega$. A point $\lambda$ with $\operatorname{Re} \lambda>\omega$ belongs to $\sigma(\mathfrak{A})$ if and only if it belongs to $\sigma(A)$, and a point $\lambda$ with $\operatorname{Re} \lambda<\omega$ belongs to $\sigma(\mathfrak{A})$ if and only if $S_{\Sigma}(\lambda)$ is not invertible. 
Proof. (i) Let $\left[\begin{array}{c}y_{0} \\ x_{0} \\ u_{0}\end{array}\right] \in \mathcal{D}(\mathfrak{A})$, and denote (for all $t \geq 0$ )

$$
\left[\begin{array}{l}
y_{t} \\
x_{t} \\
u_{t}
\end{array}\right]=\mathfrak{T}_{t}\left[\begin{array}{l}
y_{0} \\
x_{0} \\
u_{0}
\end{array}\right], \quad\left[\begin{array}{c}
\tilde{y} \\
\tilde{x} \\
\tilde{u}
\end{array}\right]=\mathfrak{A}\left[\begin{array}{l}
y_{0} \\
x_{0} \\
u_{0}
\end{array}\right] .
$$

Since the $\operatorname{limit}_{t \downarrow 0} \frac{1}{t}\left(u_{t}-u_{0}\right)=\tilde{u}$ exists in $L_{\omega}^{2}([0, \infty) ; U), u_{0}$ belongs to the domain of the generator of the left-shift semigroup $\mathbf{S}^{*}$ acting on $L_{\omega}^{2}([0, \infty)$; $U)$, i.e., $u_{0} \in H_{\omega}^{1}([0, \infty) ; U)$ and $\tilde{u}=u_{0}^{\prime}$. Since $x_{t}$ is the state trajectory of $\Sigma$ corresponding to the initial state $x_{0}$ and the input function $u_{0}$, it is in $H_{\text {loc }}^{1}\left([0, \infty), X_{-1}\right)$ and we have $\dot{x}_{t}=A x_{t}+B u_{0}(t)$ in $X_{-1}$, for almost every $t \geq 0$ (see Proposition 2.6). Since $A x_{t}+B u_{0}(t)$ is a continuous function of $t$ with values in $X_{-1}$, we conclude that $\dot{x}_{t}=A x_{t}+B u_{0}(t)$ holds (in $X_{-1}$ ) for every $t \geq 0$. Since $\left[\begin{array}{c}y_{0} \\ x_{0} \\ u_{0}\end{array}\right] \in \mathcal{D}(\mathfrak{A}), x_{t}$ is a continuously differentiable function with values in $X$. Hence, $A x_{t}+B u_{0}(t)$ is a continuous function of $t$ with values in $X$, in particular, $A x_{0}+B u_{0}(0) \in X$, so that $\left[\begin{array}{c}x_{0} \\ u_{0}(0)\end{array}\right] \in V$ and $\tilde{x}=\lim _{t \downarrow} \frac{1}{t}\left(x_{t}-x_{0}\right)=A x_{0}+B u_{0}(0)$. According to Theorem 3.1, the output $z$ of $\Sigma$ belongs to $H_{\omega}^{1}([0, \infty) ; Y)$ and satisfies $z(t)=C \& D\left[\begin{array}{c}x_{t} \\ u_{0}(t)\end{array}\right]=$ $C \& D\left[\begin{array}{c}x_{t} \\ u_{t}(0)\end{array}\right]$ for every $t \geq 0$. By the definition of $\mathfrak{T}$, we can decompose $y_{t}$ as follows:

$$
y_{t}(\eta)= \begin{cases}y_{0}(t+\eta) & \text { for } \eta<-t, \\ z(t+\eta) & \text { for }-t \leq \eta \leq 0 .\end{cases}
$$

Since $\left[\begin{array}{c}y_{0} \\ x_{0} \\ u_{0}\end{array}\right] \in \mathcal{D}(\mathfrak{A})$, the $\operatorname{limit}_{\lim _{t \downarrow}} \frac{1}{t}\left(y_{t}-y_{0}\right)=\tilde{y}$ exists in $L_{\omega}^{2}((-\infty, 0] ; Y)$. This implies that $y_{0} \in H_{\omega}^{1}((-\infty, 0] ; Y)$ and $y_{0}(0)=z(0)=C \& D\left[\begin{array}{c}x_{0} \\ u_{0}(0)\end{array}\right]$, and that the limit is $\tilde{y}=y_{0}^{\prime}$. Thus, $y_{0}, x_{0}$ and $u_{0}$ satisfy the requirements listed in point (i), and we have checked all three components of (6.2).

Conversely, suppose that $y_{0}, x_{0}$ and $u_{0}$ satisfy the requirements in point (i). Then by simple arguments using Theorem 3.1 we conclude that the three limits giving $\tilde{y}, \tilde{x}$ and $\tilde{u}$ exist, and hence $\left[\begin{array}{l}y_{0} \\ x_{0} \\ u_{0}\end{array}\right] \in \mathcal{D}(\mathfrak{A})$.

(ii) This statement is a consequence of (i) via simple algebraic manipulations.

(iii) If $\operatorname{Re} \lambda=\omega$, then $u$ given in the last part of (6.3) (with $u_{0} \in H_{\omega}^{1}([0, \infty) ; U)$ ) cannot be any function in $L_{\omega}^{2}([0, \infty) ; U)$. Indeed, the Laplace transform $\hat{u}_{0}$ given by $\hat{u}_{0}(s)=(s-\lambda)^{-1}\left(\hat{u}(s)+u_{0}(0)\right)$ must be square integrable on the line where $\operatorname{Re} s=\omega$, and this excludes functions such as $\hat{u}(s)=(s-\lambda)^{-0.3}(s-\omega+1)^{-1} \mathrm{v}$ with a nonzero $\mathrm{v} \in U$. Thus, $\lambda I-\mathfrak{A}$ is not onto, so that the vertical line where $\operatorname{Re} \lambda=\omega$ belongs to the spectrum of $\mathfrak{A}$ (proving the first statement in (iii)).

If $\operatorname{Re} \lambda>\omega$, we show that the equation $\left[\begin{array}{l}y \\ x \\ u\end{array}\right]=(\mathfrak{A}-\lambda I)\left[\begin{array}{l}y_{0} \\ x_{0} \\ u_{0}\end{array}\right]$, in the unknowns $y_{0}, x_{0}$ and $u_{0}$, has a unique solution if and only if $\lambda \in \rho(A)$. It will be more convenient to use the equivalent form (6.3) of this equation.

The equation $u=u_{0}^{\prime}-\lambda u_{0}$ (which is contained in ([6.3)) has the unique solution $u_{0}$ given via its Laplace transform by

$$
\hat{u}_{0}(s)=\frac{\hat{u}(s)-\hat{u}(\lambda)}{s-\lambda} .
$$

Observe that $u_{0}(0)=-\hat{u}(\lambda)$. We substitute this value into $(A-\lambda I) x_{0}+B u_{0}(0)=x$ (which is part of (6.3)), and observe that the resulting equation has a unique solution $x_{0}$ for all $x \in X$ if and only if $\lambda \in \rho(A)$. (For the "only if" part, notice 
that $u$ can be chosen such that $u_{0}(0)=0$.) When $\lambda \in \rho(A)$ and $x_{0}$ has been found, we can compute $y_{0}(0)$ from the first equation in (6.3). Then, $y_{0}$ can be computed by solving its differential equation $y_{0}^{\prime}-\lambda y_{0}=y$ (contained in (6.3)) with the boundary condition $y_{0}(0)$ known. We conclude that, for $\operatorname{Re} \lambda>\omega, \lambda \in \rho(\mathfrak{A})$ if and only if $\lambda \in \rho(A)$.

For $\operatorname{Re} \lambda<\omega$, again (as for $\operatorname{Re} \lambda>\omega$ ) we examine the solvability of (6.3). The equation $y_{0}^{\prime}-\lambda y_{0}=y$ (contained in (6.3)) has a unique solution given by

$$
y_{0}(t)=\int_{-\infty}^{t} e^{\lambda(t-\eta)} y(\eta) \mathrm{d} \eta,
$$

for all $t \leq 0$, in particular yielding $y_{0}(0)$. The first equation in (6.3) has a unique solution $\left[\begin{array}{c}x_{0} \\ u_{0}(0)\end{array}\right]$ in terms of $\left[\begin{array}{c}x \\ y_{0}(0)\end{array}\right]$ if and only if $S_{\Sigma}(\lambda)$ is invertible. Once we have $u_{0}(0)$, we can easily solve the differential equation $u_{0}^{\prime}-\lambda u_{0}=u$ to obtain $u_{0}$. Thus, we conclude that for $\operatorname{Re} \lambda<\omega, \lambda \in \rho(\mathfrak{A})$ if and only if $S_{\Sigma}(\lambda)$ is invertible.

The following proposition characterizes the resolvent of $\boldsymbol{A}$.

Proposition 6.4. Let $\omega \in \mathbb{R}$, let $\mathfrak{T}$ be the Lax-Phillips semigroup of index $\omega$ induced by $\Sigma$, and let $\mathfrak{A}$ be its generator. $\mathbf{G}$ is the analytic function defined on $\rho(A)$ by (3.4) (so that for $\operatorname{Re} s>\omega_{\mathbb{T}}$ it is the transfer function of $\Sigma$ ).

(i) Let $s \in \rho(\mathfrak{A})$ with $\operatorname{Re} s>\omega$ and let $\left[\begin{array}{l}y \\ x \\ u\end{array}\right] \in L_{\omega}^{2}((-\infty, 0] ; Y) \times X \times L_{\omega}^{2}([0, \infty) ; U)$. Then the following conditions are equivalent:

(a) $\left[\begin{array}{l}y_{0} \\ x_{0} \\ u_{0}\end{array}\right]=(\mathfrak{A}-s I)^{-1}\left[\begin{array}{l}y \\ x \\ u\end{array}\right]$,

$$
\left\{\begin{aligned}
{\left[\begin{array}{c}
x_{0} \\
y_{0}(0)
\end{array}\right] } & =-\left[\begin{array}{cc}
I & 0 \\
C \& D
\end{array}\right]\left[\begin{array}{cc}
s I-A & -B \\
0 & I
\end{array}\right]^{-1}\left[\begin{array}{c}
x \\
\hat{u}(s)
\end{array}\right] \\
& =-\left[\begin{array}{cc}
(s I-A)^{-1} & (s I-A)^{-1} B \\
C(s I-A)^{-1} & \mathbf{G}(s)
\end{array}\right]\left[\begin{array}{c}
x \\
\hat{u}(s)
\end{array}\right] \\
y_{0}(t) & =e^{s t} y_{0}(0)-\int_{t}^{0} e^{s(t-\eta)} y(\eta) \mathrm{d} \eta, \quad t \leq 0, \\
u_{0}(t) & =-\int_{t}^{\infty} e^{s(t-\eta)} u(\eta) \mathrm{d} \eta, \quad t \geq 0 \quad\left(\text { hence, } \quad u_{0}(0)=-\hat{u}(s)\right) .
\end{aligned}\right.
$$

(ii) Let $s \in \rho(\mathfrak{A})$ with $\operatorname{Re} s<\omega$ and let $\left[\begin{array}{l}y \\ x \\ u\end{array}\right] \in L_{\omega}^{2}((-\infty, 0] ; Y) \times X \times L_{\omega}^{2}([0, \infty) ; U)$.

Then the following conditions are equivalent:

(a) $\left[\begin{array}{l}y_{0} \\ x_{0} \\ u_{0}\end{array}\right]=(\mathfrak{A}-s I)^{-1}\left[\begin{array}{l}y \\ x \\ u\end{array}\right]$,

(b) $\left\{\begin{aligned} {\left[\begin{array}{c}x_{0} \\ u_{0}(0)\end{array}\right] } & =S_{\Sigma}(s)^{-1}\left[\begin{array}{c}x \\ y \\ (s)\end{array}\right], \\ y_{0}(t) & =\int_{-\infty}^{t} e^{s(t-\eta)} y(\eta) \mathrm{d} \eta, \quad t \leq 0, \\ u_{0}(t) & =e^{s t} u_{0}(0)+\int_{0}^{t} e^{s(t-\eta)} u(\eta) \mathrm{d} \eta, \quad t \geq 0 .\end{aligned}\right.$

The proof consists of straightforward computations based on Theorem 6.3 and its proof as well as Proposition [3.6. and we omit it. Note that $S_{\Sigma}(s)^{-1}$ can be expressed using any of the factorizations given in Proposition 3.6

We comment on the connection with the work of Lax and Phillips: Assuming $U=Y$ and $\omega=0$, we identify the unperturbed unitary group in [12, 13] with the 
left shift group $\mathcal{S}_{-t}$ on $L^{2}((-\infty, \infty) ; U)$. The spaces $\mathcal{U}$ and $\mathcal{Y}$ from Proposition 6.2 (with $\omega=0$ ) are orthogonal incoming and outgoing subspaces for $\mathcal{S}_{-t}$, respectively, and $\mathcal{F}$ from Proposition 5.3 is the scattering operator. Much useful information on how to translate scattering theory into the language of systems theory is found in [6]. We mention that in [12] and [13, in addition to the contractivity assumption on $\boldsymbol{T}$, some further controllability and observability type assumptions are made.

The early development of the theory of well-posed linear systems that took place in the Soviet Union in the eighties appears to have used the one-to-one connection between a (dissipative) well-posed linear system and a (contractive) Lax-Phillips semigroup of index zero as a starting point.

In [6, 12, 13, the operator

$$
W_{-}=\left[\begin{array}{c}
\mathbf{P}_{-} \mathcal{F} \\
\widetilde{\Phi}_{0} \\
\mathbf{P}_{+}
\end{array}\right]
$$

(denoted by very different symbols) is called the backward wave operator, and its action on exponential inputs (restricted to $(-\infty, 0])$ is investigated. Translated into our language and our somewhat different framework, the result is as follows:

Proposition 6.5. Denote the generator of $\mathbb{T}$ by $A$ and the control operator of $\Sigma$ by $B$. Then for every $u_{0} \in U$, for all $\lambda \in \mathbb{C}_{\omega_{\mathbb{T}}}$ and for all $t \in \mathbb{R}$,

$$
\begin{aligned}
\widetilde{\Phi}_{t}\left(e_{\lambda} u_{0}\right) & =e^{\lambda t}(\lambda I-A)^{-1} B u_{0}, \\
\mathcal{F}\left(e_{\lambda} u_{0}\right) & =e_{\lambda} \mathbf{G}(\lambda) u_{0},
\end{aligned}
$$

where $e_{\lambda}$ is the function $e_{\lambda}(t)=e^{\lambda t}$, for all $t \in \mathbb{R}$.

Proof. To prove (6.4), we substitute $u=e_{\lambda} u_{0}$ in (5.2) to get

$$
\begin{aligned}
\widetilde{\Phi}_{t}\left(e_{\lambda} u_{0}\right) & =\int_{-\infty}^{0} e^{\lambda(\sigma+t)} \mathbb{T}_{-\sigma} B u_{0} \mathrm{~d} \sigma=e^{\lambda t} \int_{0}^{\infty} e^{-\lambda \sigma} \mathbb{T}_{\sigma} B u_{0} \mathrm{~d} \sigma \\
& =e^{\lambda t}(\lambda I-A)^{-1} B u_{0}
\end{aligned}
$$

Let $\omega, T \in \mathbb{R}$ with $\omega_{\mathbb{T}}<\omega<\operatorname{Re} \lambda$, and choose $u \in H_{\omega}^{1}((-\infty, \infty) ; U)$ such that $\mathbf{P}_{(-\infty, T]} u=e_{\lambda} u_{0}$. Denote $y=\mathcal{F} u$. By causality, $\mathbf{P}_{(-\infty, T]} y=\mathbf{P}_{(-\infty, T]} \mathcal{F}\left(e_{\lambda} u_{0}\right)$. Denote $x(t)=\widetilde{\Phi}_{t} u$, so that for $t \leq T, x(t)=e^{\lambda t}(\lambda I-A)^{-1} B u_{0}$. By Theorem 5.6)(ii) and the formula (3.4) for $\mathbf{G}$, for all $t \in(-\infty, T]$,

$$
y(t)=C \& D\left[\begin{array}{l}
x(t) \\
u(t)
\end{array}\right]=e^{\lambda t} C \& D\left[\begin{array}{c}
(\lambda I-A)^{-1} B \\
I
\end{array}\right] u_{0}=e^{\lambda t} \mathbf{G}(\lambda) u_{0} .
$$

Since $T \in \mathbb{R}$ was arbitrary, we get that (6.5) holds.

Remark 6.6. The last proposition is not stated in the most general form. Indeed, if $\gamma_{\mathbb{F}}<\omega_{\mathbb{T}}$, where $\gamma_{\mathbb{F}}$ is the growth bound of $\mathbb{F}_{\infty}$, then formula (6.5) remains valid on the larger half-plane $\lambda \in \mathbb{C}_{\gamma_{\mathbb{F}}}$. The most concise argument for this is to regard both sides as analytic functions defined on $\mathbb{C}_{\omega_{\mathbb{T}}}$ with values in the Fréchet space $L_{\omega, \text { loc }}^{2}((-\infty, \infty) ; Y)$, where $\omega \in\left(\gamma_{\mathbb{F}}, \omega_{\mathbb{T}}\right]$. Both sides have analytic extensions to $\mathbb{C}_{\omega}$, and hence these extensions must be equal on $\mathbb{C}_{\omega}$. Since $\omega \in\left(\gamma_{\mathbb{F}}, \omega_{\mathbb{T}}\right]$ was arbitrary, we get equal analytic extensions on $\mathbb{C}_{\gamma_{\mathbb{F}}}$, meaning that 6.5 holds on $\mathbb{C}_{\gamma_{\mathbb{F}}}$.

In the scattering theory of Lax and Phillips [12,13] (and also in [36]) the identity (6.5) is taken as the definition of $\mathbf{G}(\lambda)$, which is called the scattering matrix in that 
context. We refer to Staffans [35] or [36] for further discussions of the connection between scattering theory and the theory of well-posed linear systems.

Recall that if $u \in L_{\omega}^{2}([0, \infty) ; U)$ with $\omega>\gamma_{\mathbb{F}}$, then the Laplace transform of $\mathbb{F}_{\infty} u$ is given by $\widehat{\mathbb{F}_{\infty} u}(s)=\mathbf{G}(s) \hat{u}(s)$ for all $s \in \mathbb{C}_{\omega}$. A similar result is valid with $\mathbb{F}_{\infty}$ replaced by $\mathcal{F}$. To state this result, we need the bilateral Laplace transform:

Definition 6.7. Let $W$ be a Hilbert space. The bilateral Laplace transform of a function $u \in L_{\text {loc }}^{2}((-\infty, \infty) ; W)$ is defined by $\hat{u}(s)=\int_{-\infty}^{\infty} e^{-s t} u(t) \mathrm{d} t$, for all those $s \in \mathbb{C}$ for which the integral converges absolutely.

Clearly, if $u(t)=0$ for $t<0$, then we recover the usual Laplace transform. It is easy to see that the domain of definition of $\hat{u}$, if nonempty, is a vertical strip $\{s \in \mathbb{C} \mid \operatorname{Re} s \in J\}$, where $J$ is an interval in $\mathbb{R}$. In the interior of this vertical strip $\hat{u}$ is analytic. The interval $J$ may be unbounded, at each end it may be open or closed, and it may consist of a single point (see [3, 23]). If $u \in L_{\omega}^{2}((-\infty, \infty) ; W)$ and if $J$ is nonempty, then $\omega \in \bar{J}$. If it happens that $\omega \in J$, i.e., if $\hat{u}$ is defined on the line where $\operatorname{Re} s=\omega$, then the function $\sigma \mapsto \hat{u}(\omega+i \sigma),-\infty<\sigma<\infty$, can be interpreted as $\sqrt{2 \pi}$ times the Fourier transform of the function $e_{-\omega} u$, and Parseval's identity gives

$$
\int_{-\infty}^{\infty}\left\|e^{-\omega t} u(t)\right\|^{2} \mathrm{~d} t=\frac{1}{2 \pi} \int_{-\infty}^{\infty}\|\hat{u}(\omega+i \sigma)\|^{2} \mathrm{~d} \sigma .
$$

The above fact motivates us to slightly extend the definition of the bilateral Laplace transform. We define the bilateral Laplace transform of a function $u \in$ $L_{\omega}^{2}((-\infty, \infty) ; W)$ on the vertical line $\operatorname{Re} s=\omega$ to be $\sqrt{2 \pi}$ times the (almost everywhere defined) Fourier transform of $e_{-\omega} u$ (in the $L^{2}$ sense). We still denote this transform by $\hat{u}(\omega+i \sigma),-\infty<\sigma<\infty$, even if $\omega \notin J$. If $\omega \notin J$ but $\omega \in \bar{J}$, then for almost every $\sigma \in \mathbb{R}, \hat{u}(\omega+i \sigma)$ is the nontangential limit of $\hat{u}(s)$ with $\operatorname{Re} s \in J$ (this follows from well-known facts about Hardy spaces).

Proposition 6.8. Let $\omega>\gamma_{\mathbb{F}}$ and $u \in L_{\omega}^{2}((-\infty, \infty) ; U)$. Then

$$
\widehat{\mathcal{F} u}(s)=\mathbf{G}(s) \hat{u}(s)
$$

for almost every $s \in \mathbb{C}$ with $\operatorname{Re} s=\omega$. (This means that, for almost every $\sigma \in \mathbb{R}$, (6.6) holds with $s=\omega+i \sigma$.) If $\mathbf{P}_{+} u \in L_{\alpha}^{2}([0, \infty) ; U)$ and $\mathbf{P}_{-} u \in L_{\beta}^{2}((-\infty, 0] ; U)$ with $\gamma_{\mathbb{F}}<\alpha<\beta$, then (6.6) holds for all $s \in \mathbb{C}$ with $\alpha<\operatorname{Re} s<\beta$ and for almost every $s \in \mathbb{C}$ with $\operatorname{Re} s=\alpha$ or $\operatorname{Re} s=\beta$.

Proof. Take $\nu \in\left(\gamma_{\mathbb{F}}, \omega\right)$. If $u$ has compact support contained in $[0, \infty)$, then $u \in$ $L_{\nu}^{2}([0, \infty) ; U)$, and (6.6) holds for all $s \in \mathbb{C}_{\nu}$ because of (2.19) and the fact that $\mathcal{F} \mathbf{P}_{+}=\mathbb{F}_{\infty}$. If $u$ has compact support contained in $[-\tau, \infty)$, then by the causality of $\mathcal{F}$, the supports of $\mathcal{S}_{\tau} u$ and $\mathcal{S}_{\tau} \mathcal{F} u$ are contained in $[0, \infty)$. Therefore we can apply the preceding result, which together with the shift-invariance of $\mathcal{F}$ gives

$$
e^{-\tau s} \widehat{\mathcal{F} u}(s)=\widehat{\mathcal{S}_{\tau} \mathcal{F} u}(s)=\widehat{\mathcal{F} \mathcal{S}_{\tau} u}(s)=\mathbf{G}(s) \widehat{\mathcal{S}_{\tau} u}(s)=e^{-\tau s} \mathbf{G}(s) \widehat{u}(s)
$$

for all $s \in \mathbb{C}$ with $\operatorname{Re} s>\nu$. In particular, (6.6) holds for $\operatorname{Re} s=\omega$ in this case, too. Regarded as elements of $L^{2}$ of the vertical line where $\operatorname{Re} s=\omega$, both sides of (6.6) depend continuously on $u \in L_{\omega}^{2}(((-\infty, \infty) ; U)$. Since functions with compact support are dense in $L_{\omega}^{2}((-\infty, \infty) ; U)$, we can extend (6.6) by continuity (as an identity in $\left.L^{2}\right)$ to all functions $u \in L_{\omega}^{2}((-\infty, \infty) ; U)$. Being an identity in $L^{2}$, the formula now holds only almost everywhere. 
Suppose that the conditions in the second part of the proposition hold. Then, by elementary estimates, we find that $u \in L_{\nu}^{2}((-\infty, \infty) ; U)$ and $\mathcal{F} u \in L_{\nu}^{2}((-\infty, \infty) ; Y)$ for all $\nu \in[\alpha, \beta]$, and $u \in L_{\nu}^{1}((-\infty, \infty) ; U)$ and $\mathcal{F} u \in L_{\nu}^{1}((-\infty, \infty) ; Y)$ for all $\nu \in(\alpha, \beta)$. By the first part of the proposition, (6.6) holds almost everywhere on each vertical line $\operatorname{Re} s=\nu$ with $\nu \in[\alpha, \beta]$. Since both sides are analytic in the strip $\alpha<\operatorname{Re} s<\beta$, we must have equality everywhere in this strip.

We mention that for some systems, (6.6) remains valid for almost every $s \in \mathbb{C}$ with $\operatorname{Re} s=\omega$, even if $\omega=\gamma_{\mathbb{F}}$. See the discussion in Thomas [37].

\section{Dissipative Systems}

In this section we briefly discuss dissipative well-posed linear systems, mainly the characterization of such systems. We use the same notation as in Section 6 ] In particular, the system $\Sigma=(\mathbb{T}, \Phi, \Psi, \mathbb{F})$ as well as the spaces $U, X, Y, V$ and the operators $\tilde{\Phi}_{t}, \Psi_{\infty}, \mathbb{F}_{\infty}, \mathcal{F}, A, B, C, C \& D$ are as in Notation 6.1 The system operator of $\Sigma$ is $S_{\Sigma}(s)$ and the growth bound of $\mathbb{T}$ is $\omega_{\mathbb{T}}$. The function $\mathbf{G}$ is defined on $\rho(A)$ by (3.4) (as in Proposition 6.4). Thus, for $\operatorname{Re} s>\omega_{\mathbb{T}}, \mathbf{G}(s)$ is the transfer function of $\Sigma$ (see also the comments before Proposition 3.6).

Definition 7.1. The system $\Sigma$ is called dissipative if for all $x_{0} \in X$ and all $u \in$ $L^{2}([0, \infty) ; U)$, the following holds: if $x$ is the state trajectory and $y$ is the output function of $\Sigma$, as in (2.14), then for all $t \geq 0$,

$$
\|x(t)\|^{2}-\left\|x_{0}\right\|^{2} \leq \int_{0}^{t}\|u(\sigma)\|^{2} \mathrm{~d} \sigma-\int_{0}^{t}\|y(\sigma)\|^{2} \mathrm{~d} \sigma .
$$

The following proposition shows the connection between the dissipativity of $\Sigma$ and the Lax-Phillips semigroup induced by $\Sigma$.

Proposition 7.2. The following conditions are equivalent:

(i) $\Sigma$ is dissipative.

(ii) For every $\tau>0$, the operator $\Sigma_{\tau}=\left[\begin{array}{ll}\mathbb{T}_{\tau} & \Phi_{\tau} \\ \Psi_{\tau} & \mathbb{F}_{\tau}\end{array}\right]$ is a contraction from $X \times$ $L^{2}([0, \tau] ; U)$ to $X \times L^{2}([0, \tau] ; Y)$.

(iii) The Lax-Phillips semigroup of index zero induced by $\Sigma$ is contractive.

Moreover, if $\Sigma$ is dissipative, then its semigroup $\mathbb{T}$ is contractive, we have

$$
\begin{gathered}
\widetilde{\Phi}_{0} \in \mathcal{L}\left(L^{2}((-\infty, 0] ; U) ; X\right), \quad \Psi_{\infty} \in \mathcal{L}\left(X ; L^{2}([0, \infty) ; Y),\right. \\
\mathcal{F} \in \mathcal{L}\left(L^{2}((-\infty, \infty) ; U) ; L^{2}((-\infty, \infty) ; Y)\right.
\end{gathered}
$$

and these three operators are contractions.

Note that in (ii) we use the norm $\left\|\left[\begin{array}{c}x_{0} \\ u\end{array}\right]\right\|^{2}=\left\|x_{0}\right\|^{2}+\|u\|_{L^{2}([0, \tau] ; U)}^{2}$ on $X \times$ $L^{2}([0, \tau] ; U)$ and the analogous norm on $X \times L^{2}([0, \tau] ; Y)$. Note also that (7.3) implies that $\mathbb{F}_{\infty}$ is a contraction from $L^{2}([0, \infty) ; U)$ to $L^{2}([0, \infty) ; Y)$. According to the end of Section 2, this implies $\sup _{s \in \mathbb{C}_{0}}\|\mathbf{G}(s)\| \leq 1$. This will be further refined in Remark 7.5

Proof. (i) $\Leftrightarrow$ (ii): This follows from Definitions 2.4 and 7.1, with (1.5)).

(ii) $\Leftrightarrow$ (iii): Let $y_{0} \in L^{2}((-\infty, 0] ; Y), x_{0} \in X, u_{0} \in L^{2}([0, \infty) ; U)$, and let $x$ be the state trajectory and $y$ the output function of $\Sigma$, as in (2.14) (with $u_{0}$ in place 
of $u$ ). We denote by $\boldsymbol{T}$ the Lax-Phillips semigroup of index zero induced by $\Sigma$, as in Proposition 6.2 According to (6.1), for all $t \geq 0$,

$$
\begin{aligned}
\left\|\mathfrak{T}_{t}\left[\begin{array}{c}
y_{0} \\
x_{0} \\
u_{0}
\end{array}\right]\right\|^{2}-\left\|\left[\begin{array}{c}
y_{0} \\
x_{0} \\
u_{0}
\end{array}\right]\right\|^{2} & =\int_{-\infty}^{0}\left\|y_{0}(\sigma)\right\|^{2} \mathrm{~d} \sigma+\int_{0}^{t}\|y(\sigma)\|^{2} \mathrm{~d} \sigma \\
& +\|x(t)\|^{2}+\int_{t}^{\infty}\left\|u_{0}(\sigma)\right\|^{2} \mathrm{~d} \sigma \\
& -\int_{-\infty}^{0}\left\|y_{0}(\sigma)\right\|^{2} \mathrm{~d} \sigma-\left\|x_{0}\right\|^{2}-\int_{0}^{\infty}\left\|u_{0}(\sigma)\right\|^{2} \mathrm{~d} \sigma \\
& =\int_{0}^{t}\|y(\sigma)\|^{2} \mathrm{~d} \sigma+\|x(t)\|^{2}-\left\|x_{0}\right\|^{2}-\int_{0}^{t}\left\|u_{0}(\sigma)\right\|^{2} \mathrm{~d} \sigma .
\end{aligned}
$$

The left-hand side above is $\leq 0$ for all $y_{0}, x_{0}$, and $u_{0}$ if and only if $\mathfrak{T}$ is contractive, and the right-hand side is $\leq 0$ for all $x_{0}$ and $u_{0}$ if and only if $\Sigma$ is dissipative, so that $\mathfrak{T}$ is contractive if and only if $\Sigma$ is dissipative.

The final claims are consequences of (ii). Indeed, to show the first part of (7.2), we use that for all $v \in L^{2}((-\infty, 0] ; U)$ and for $0 \leq \tau \leq T$ we have

$$
\left\|\Phi_{T} \mathcal{S}_{T} v-\Phi_{\tau} \mathcal{S}_{\tau} v\right\| \leq\left\|\mathbb{T}_{\tau}\right\| \cdot\left\|\Phi_{T-\tau} \mathcal{S}_{T} v\right\| .
$$

This is proved exactly as (5.5) (with $\omega=0$ and $t=0$ ). Since, by (ii), $\mathbb{T}_{\tau}$ and $\Phi_{T-\tau}$ are contractions (and $\mathcal{S}_{T}$ is also a contraction), we obtain $\left\|\Phi_{T} \mathcal{S}_{T} v-\Phi_{\tau} \mathcal{S}_{\tau} v\right\| \leq$ $\left\|\mathbf{P}_{[-T,-\tau]} v\right\|_{L^{2}}$. This proves that the limit (5.1) (with $t=0$ ) exists and, by taking $\tau=0$ and $T \rightarrow \infty$, it shows that $\widetilde{\Phi}_{0}$ is a contraction. The proof of the second part of (7.2) is much easier. The proof of (7.3) is similar to the proof of the first part of (7.2), by slightly modifying a part of the proof of Proposition 5.3.

We denote by $B C^{1}([0, \infty) ; X)$ the space of all the bounded functions contained in $C^{1}([0, \infty) ; X)$ whose derivatives are also bounded.

Proposition 7.3. Suppose that $\Sigma$ is dissipative, $x_{0} \in X$ and $u \in H^{1}([0, \infty) ; U)$ are such that $\left[\begin{array}{c}x_{0} \\ u(0)\end{array}\right] \in V$. If $x$ is the state trajectory and $y$ is the output function corresponding to $x_{0}$ and $u$ (as in (2.14)), then

$$
x \in B C^{1}([0, \infty) ; X), \quad y \in H^{1}([0, \infty) ; Y) .
$$

Proof. According to Theorem 3.1 (i), $x$ is continuously differentiable on $[0, \infty)$ and $y \in H_{\omega}^{1}([0, \infty) ; Y)$ for all $\omega>0$. The boundedness of the state trajectory $x$ follows from the fact that the operators $\mathbb{T}_{\tau}$ and $\Phi_{\tau}$ are uniformly bounded. The boundedness of its derivative follows similarly, from the formula

$$
\dot{x}(t)=\mathbb{T}_{t}\left(A x_{0}+B u(0)\right)+\Phi_{t} \dot{u} .
$$

To obtain that $y \in H^{1}([0, \infty) ; Y)$, we have to modify slightly the last part of the proof of Theorem 3.1. Indeed, using the second part of (7.2) and (7.3), we see that (3.6) holds for $\omega=0$.

For the following theorem we need the spaces $X_{1}^{d}$ and $X_{-1}^{d}$, which are similar to $X_{1}$ and $X_{-1}$. The space $X_{1}^{d}$ is $\mathcal{D}\left(A^{*}\right)$ with the norm $\|z\|_{1}^{d}=\left\|\left(\beta I-A^{*}\right) z\right\|$, where $\beta \in \rho\left(A^{*}\right)$, while $X_{-1}^{d}$ is defind as the completion of $X$ with respect to the norm $\|z\|_{-1}^{d}=\left\|\left(\beta I-A^{*}\right)^{-1} z\right\|$. It is easy to see that $X_{-1}^{d}$ is isomorphic to the dual of $X_{1}$ with respect to the pivot space $X$. We identify $U$ and $Y$ with their duals, so that $B^{*} \in \mathcal{L}\left(X_{1}^{d} ; U\right)$ and $C^{*} \in \mathcal{L}\left(Y ; X_{-1}^{d}\right)$. If $T \in \mathcal{L}\left(X_{1} ; X_{-1}^{d}\right)$, then also $T^{*} \in \mathcal{L}\left(X_{1} ; X_{-1}^{d}\right)$. For such a $T$, we say that $T \leq 0$ if $T^{*}=T$ and $\langle T z, z\rangle \leq 0$ for 
all $z \in X_{1}$. For an operator $T \in \mathcal{L}\left(X_{1} \times U ; X_{-1}^{d} \times U\right)$, the meaning of $T \leq 0$ is similar.

Theorem 7.4. The following conditions are equivalent:

(i) $\Sigma$ is dissipative.

(ii) For all $x_{0} \in X$ and $u \in H^{1}([0, \infty) ; U)$ satisfying $\left[\begin{array}{c}x_{0} \\ u(0)\end{array}\right] \in V$, the corresponding state trajectory $x$ and output function $y$ (as in (2.14) ) satisfy

$$
\frac{\mathrm{d}}{\mathrm{d} t}\|x(t)\|^{2} \leq\|u(t)\|^{2}-\|y(t)\|^{2}, \quad \text { for all } t \geq 0 .
$$

(iii) For all $\left[\begin{array}{l}x \\ u\end{array}\right] \in V$,

$$
2 \operatorname{Re}\langle A x+B u, x\rangle \leq\|u\|^{2}-\left\|C \& D\left[\begin{array}{l}
x \\
u
\end{array}\right]\right\|^{2} .
$$

(iv) For some $s \in \rho(A)$ we have

$$
\begin{gathered}
{\left[\begin{array}{cc}
A+A^{*} & \left(s I+A^{*}\right)(s I-A)^{-1} B \\
B^{*}\left(\bar{s} I-A^{*}\right)^{-1}(\bar{s} I+A) & B^{*}\left(\bar{s} I-A^{*}\right)^{-1}(2 \operatorname{Re} s)(s I-A)^{-1} B
\end{array}\right]} \\
+\left[\begin{array}{cc}
C^{*} C & C^{*} \mathbf{G}(s) \\
\mathbf{G}(s)^{*} C & \mathbf{G}(s)^{*} \mathbf{G}(s)
\end{array}\right] \leq\left[\begin{array}{ll}
0 & 0 \\
0 & I
\end{array}\right],
\end{gathered}
$$

which is an operator inequality in $\mathcal{L}\left(X_{1} \times U ; X_{-1}^{d} \times U\right)$.

(v) The inequality (7.6) holds for all $s \in \rho(A)$.

(vi) For some $s \in \rho(A) \cap \mathbb{C}_{0}$, the operator

$$
\left[\begin{array}{ll}
\mathbf{A}(s) & \mathbf{B}(s) \\
\mathbf{C}(s) & \mathbf{G}(s)
\end{array}\right]=\left[\begin{array}{cc}
(\bar{s} I+A)(s I-A)^{-1} & \sqrt{2 \operatorname{Re} s}(s I-A)^{-1} B \\
\sqrt{2 \operatorname{Re} s} C(s I-A)^{-1} & \mathbf{G}(s)
\end{array}\right]
$$

is a contraction from $X \times U$ to $X \times Y$.

(vii) $\mathbb{C}_{0} \subset \rho(A)$, and for all $s \in \mathbb{C}_{0}$, the operator $\left[\begin{array}{ll}\mathbf{A}(s) & \mathbf{B}(s) \\ \mathbf{C}(s) & \mathbf{G}(s)\end{array}\right]$ defined in (7.7) is a contraction from $X \times U$ to $X \times Y$.

Proof. (i) $\Rightarrow$ (ii): We know from Theorem 3.1(i) (or from Proposition 7.3) that $x$ is continuously differentiable and $u, y$ are continuous. To obtain (7.4), we differentiate (7.1) with respect to $t$ (we use the right derivative at $t=0$ ).

(ii) $\Rightarrow$ (i): If (ii) holds then, whenever $x_{0}$ and $u$ satisfy the conditions listed in (ii), we find that (7.1) holds (by integrating (7.4)). The sides of (7.1) depend continuously on $x_{0}$ and $u$. Since the set of possible $x_{0}$ and $u$ in (ii) is dense in $X \times L^{2}([0, \infty) ; U)$, this implies that (7.1) holds for all $x_{0} \in X$ and all $u \in$ $L^{2}([0, \infty) ; U)$.

(ii) $\Rightarrow$ (iii): By Theorem 3.1 (i), it follows from (ii) that (7.5) holds with $\left[\begin{array}{l}x \\ u\end{array}\right]$ replaced by $\left[\begin{array}{l}x(t) \\ u(t)\end{array}\right]$ for all $t \geq 0$ (because $\left.\frac{\mathrm{d}}{\mathrm{d} t}\|x(t)\|^{2}=2 \operatorname{Re}\langle\dot{x}(t), x(t)\rangle\right)$. In particular, taking $t=0$, we get (7.5) for all $\left[\begin{array}{l}x \\ u\end{array}\right] \in V$.

(iii) $\Rightarrow$ (ii): If (iii) holds, and if $x_{0}$ and $u$ satisfy the conditions in (ii), then by Theorem 3.1 (i), $\left[\begin{array}{l}x(t) \\ u(t)\end{array}\right] \in V$ and $\left[\begin{array}{l}\dot{x}(t) \\ y(t)\end{array}\right]=\left[\begin{array}{ll}A & B \\ C \& D\end{array}\right]\left[\begin{array}{l}x(t) \\ u(t)\end{array}\right]$, so that (7.4) holds.

(iii) $\Rightarrow(\mathrm{v})$ : It follows from Proposition 3.6 that, for all $s \in \rho(A),\left[\begin{array}{l}I(s I-A)^{-1} B \\ 0\end{array}\right]$ maps $X_{1} \times U$ one-to-one onto $V$. Therefore we can replace $\left[\begin{array}{l}x \\ u\end{array}\right] \in V$ in (iii) by $\left[\begin{array}{cc}I \\ 0 & (s I-A)^{-1} B\end{array}\right]\left[\begin{array}{l}z \\ v\end{array}\right]$, where $\left[\begin{array}{c}z \\ v\end{array}\right] \in X_{1} \times U$. Doing the substitution and rearranging 
terms, we see that (7.5) becomes (7.6) (the first term in (7.6) corresponds to the term $2 \operatorname{Re}\langle A x+B u, x\rangle$ in (7.5), the second term in (7.6) corresponds to the term $\left\|C \& D\left[\begin{array}{l}x \\ u\end{array}\right]\right\|^{2}$ in (7.5), and the right-hand side of (7.6) corresponds to $\left.\|u\|^{2}\right)$.

(v) $\Rightarrow$ (iv) $\Rightarrow$ (iii): It is obvious that (v) implies (iv), and (iv) implies (iii) on multiplying (7.6) by $\left[\begin{array}{c}I(A-s I)^{-1} B \\ 0\end{array}\right]$ from the right and by its adjoint from the left. This is the computation from (iii) $\Rightarrow(\mathrm{v})$ done backwards.

(vii) $\Rightarrow(\mathrm{vi}) \Rightarrow(\mathrm{iv})$ : The first of these implications is obvious. Statement (vi) means that for some $s \in \rho(A) \cap \mathbb{C}_{0}$,

$$
\left[\begin{array}{ll}
\mathbf{A}(s) & \mathbf{B}(s) \\
\mathbf{C}(s) & \mathbf{G}(s)
\end{array}\right]^{*}\left[\begin{array}{ll}
\mathbf{A}(s) & \mathbf{B}(s) \\
\mathbf{C}(s) & \mathbf{G}(s)
\end{array}\right] \leq\left[\begin{array}{ll}
I & 0 \\
0 & I
\end{array}\right]
$$

By substituting the definitions of $\mathbf{A}(s), \mathbf{B}(s), \mathbf{C}(s)$ given in (7.7) into this inequality and simplifying the resulting expression, we obtain that

$$
\left[\begin{array}{ll}
\mathbf{M}(s) & \mathbf{N}(s) \\
\mathbf{N}^{*}(s) & \mathbf{Q}(s)
\end{array}\right] \leq\left[\begin{array}{ll}
0 & 0 \\
0 & I
\end{array}\right]
$$

where

$$
\begin{gathered}
\mathbf{M}(s)=(2 \operatorname{Re} s)\left(\bar{s} I-A^{*}\right)^{-1}\left(A+A^{*}+C^{*} C\right)(s I-A)^{-1}, \\
\mathbf{N}(s)=\sqrt{2 \operatorname{Re} s}\left(\bar{s} I-A^{*}\right)^{-1}\left(s I+A^{*}\right)(s I-A)^{-1} B+\sqrt{2 \operatorname{Re} s}\left(\bar{s} I-A^{*}\right)^{-1} C^{*} \mathbf{G}(s), \\
\mathbf{Q}(s)=(2 \operatorname{Re} s) B^{*}\left(\bar{s} I-A^{*}\right)^{-1}(s I-A)^{-1} B+\mathbf{G}(s)^{*} \mathbf{G}(s) .
\end{gathered}
$$

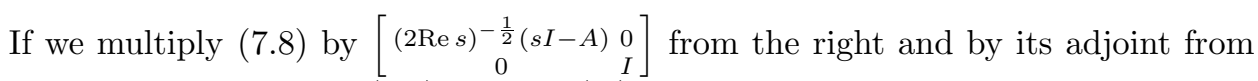
the left, we get exactly (17.6), so that (iv) holds.

(iv) $\Rightarrow$ (vii): If (iv) holds, then (by the equivalence of (i) and (iv), proved earlier), $\Sigma$ is dissipative, so that (by Proposition 7.2 ) $\mathbb{T}$ is a contraction semigroup. This implies that $\mathbb{C}_{0} \subset \rho(A)$. By the equivalence of (iv) and (v) (proved earlier), (77.6) holds for every $s \in \mathbb{C}_{0}$. Doing the steps from the proof of (vi) $\Rightarrow$ (iv) in reverse order, we obtain that (vii) holds.

Remark 7.5. The left upper corner of (7.6) implies that $A+A^{*} \leq-C^{*} C$. Similarly, the right lower corner of (7.6) implies that for $\operatorname{Re} s>0, \mathbf{G}(s)^{*} \mathbf{G}(s) \leq I$. Moreover, if $\mathrm{v} \in U$ is such that $B \mathrm{v} \neq 0$, then $\|\mathbf{G}(s) \mathrm{v}\|<\|\mathrm{v}\|$.

Remark 7.6. Suppose that $B$ is bounded, i.e., $B \in \mathcal{L}(U ; X)$ (so that, in particular, $\Sigma$ is regular) and denote the feedthrough operator of $\Sigma$ by $D$. Then $\Sigma$ is dissipative if and only if the inequality (1.8) holds. (In the context of Section 1 this inequality contained matrices, while now it contains operators. The meaning of $\leq 0$ is as explained before Theorem 7.4) Indeed, it can be checked that (1.8) is equivalent to (7.5), since now $V=X_{1} \times U$ and $C \& D\left[\begin{array}{l}x \\ u\end{array}\right]=C x+D u$. Now Theorem 7.4 (the equivalence of (i) and (iii)) shows that (1.8) is equivalent to $\Sigma$ being dissipative. We remark that (1.8) can be derived also from (7.6) by taking limits as $s \rightarrow+\infty$.

Suppose now that $C$ is bounded, i.e., $C \in \mathcal{L}(X ; Y)$ (but we do not assume $B$ to be bounded). Then we can apply the above reasoning to the dual system $\Sigma^{d}$, as defined for example in Weiss ${ }^{2}$ [5]. The control operator of $\Sigma^{d}$ is $C^{*}$, which is bounded, so that the characterization (1.8) of dissipativity holds for $\Sigma^{d}$. It is easy to see that $\Sigma^{d}$ is dissipative if and only if $\Sigma$ is dissipative. This implies that $\Sigma$ is 
dissipative if and only if

$$
\left[\begin{array}{cc}
A^{*}+A+B B^{*} & C^{*}+B D^{*} \\
C+D B^{*} & D D^{*}-I
\end{array}\right] \leq 0
$$

in the space $\mathcal{L}\left(X_{1}^{d} \times Y ; X_{-1} \times Y\right)$.

Remark 7.7. The $2 \times 2$ operator matrix appearing in (7.7) may be regarded as the system operator (evaluated at zero) of a discrete-time system with the same input, state and output spaces as $\Sigma$. (This concept has not been introduced here, but its meaning is straightforward.) The discrete-time system mentioned above is called the Cayley transform of $\Sigma$. In particular, the operator $\mathbf{A}(s)$ is the Cayley transform of $A$. In the literature, often the choice $s=1$ is considered.

Remark 7.8. Most of the results in this section are also found in Arov and Nudelman [1, but our proofs are much more direct than those given in [1, which are based on a reduction to the corresponding discrete-time results via the Cayley transform. (The paper [1] contains much additional material on dissipative and conservative systems.) Proposition 7.2 is mentioned in [1, Remark 4.1], and the reader is referred to earlier work by Adamjan, Arov and Shmulyan for its proof (see also [1. Propositions 4.2 and 4.4]). Arov and Nudelman use condition (ii) in Theorem 7.4 as the definition of dissipativity (which they call passivity), and the equivalence of conditions (ii) and (iii) in Theorem 7.4 is proved in [1, Proposition 4.1]. Via the Cayley transform, Arov and Nudelman also arrive at condition (vi) in Theorem $\mathbf{7 . 4}$ with $s=1$ (see Theorem 5.2 in [1] and the comment following its proof).

\section{ACKNOWLEDGMENT}

This research has been funded in part by the EPSRC grant no. GR/R05048/01, "Analysis and control of Lagrangian systems" (principal investigator: David J.N. Limebeer from Imperial College).

\section{REFERENCES}

[1] D.Z. Arov and M.A. Nudelman. Passive linear stationary dynamical scattering systems with continuous time. Integral Equations and Operator Theory, 24:1-45, 1996. MR 96k:47016

[2] G. Avalos, I. Lasiecka, and R. Rebarber. Lack of time-delay robustness for stabilization of a structural acoustics model. SIAM J. Control and Optimization, 37:1394-1418, 2000. MR 2000e:93064

[3] G. Doetsch. Handbuch der Laplace Transformation, Band I. Birkhäuser Verlag, Basel, 1950. MR 13:230f

[4] K.-J. Engel. On the characterization of admissible control- and observation operators. Systems and Control Letters, 34:225-227, 1998. MR 99d:93007

[5] P. Grabowski and F.M. Callier. Admissible observation operators. Semigroup criteria of admissibility. Integral Equat. \& Operator Theory, 25:182-198, 1996. MR 97d:93011

[6] J.W. Helton. Systems with infinite-dimensional state space: the Hilbert space approach. Proceedings of the IEEE, 64:145-160, 1976. MR 54:4764

[7] E. Hille and R.S. Phillips. Functional Analysis and Semi-Groups. American Mathematical Society, Providence, Rhode Island, revised edition, 1957. MR 19:664d

[8] D. Hinrichsen and A. J. Pritchard. Robust stability of bilinear evolution operators on Banach spaces. SIAM J. Control Optim., 32:1503-1541, 1994. MR 95i:93109

[9] B. Jacob and J.R. Partington. The Weiss conjecture on admissibility of observation operators for contraction semigroups. Integral Equations and Operator Theory, 40:231-243, 2001.

[10] B. Jacob and H. Zwart. Realization of inner functions. Preprint, Twente, 1998.

[11] V. Katsnelson and G. Weiss. A counterexample in Hardy spaces with an application to systems theory. Zeitschrift für Analysis und ihre Anwendungen, 14:705-730, 1995. MR 96m:47124 
[12] P.D. Lax and R.S. Phillips. Scattering Theory. Academic Press, New York, 1967. MR 36:530

[13] P.D. Lax and R.S. Phillips. Scattering theory for dissipative hyperbolic systems. J. Functional Analysis, 14:172-235, 1973. MR 50:5502

[14] H. Logemann, R. Rebarber, and G. Weiss. Conditions for robustness and nonrobustness of the stability of feedback systems with respect to small delays in the feedback loop. SIAM J. Control and Optim., 34:572-600, 1996. MR 97c:93073

[15] H. Logemann and E.P. Ryan. Time-varying and adaptive integral control of infinitedimensional regular linear systems with input nonlinearities. SIAM J. Control and Optimization, 38:1120-1144, 2000. MR 2002c:93137

[16] H. Logemann, E.P. Ryan, and S. Townley. Integral control of infinite-dimensional linear systems subject to input saturation. SIAM J. Control and Optimization, 36:1940-1961, 1998. MR 99f: 93078

[17] H. Logemann, E.P. Ryan, and S. Townley. Integral control of linear systems with actuator nonlinearities: lower bounds for the maximal regulating gain. IEEE Trans. Autom. Control, 44:1315-1319, 1999. CMP 99:14

[18] H. Logemann and S. Townley. Discrete-time low-gain control of uncertain infinite-dimensional systems. IEEE Trans. Autom. Control, 42:22-37, 1997. MR 98a:93034

[19] H. Logemann and S. Townley. Low gain control of uncertain regular linear systems. SIAM J. Control and Optimization, 35:78-116, 1997. MR 97m:93048

[20] K.A. Morris. Justification of input-output methods for systems with unbounded control and observation. IEEE Trans. Autom. Control, 44:81-84, 1999. MR 99j:93083

[21] R. Ober and S. Montgomery-Smith. Bilinear transformation of infinite-dimensional statespace systems and balanced realizations of nonrational transfer functions. SIAM J. Control and Optimization, 28:438-465, 1990. MR 91d:93019

[22] R. Ober and Y. Wu. Infinite-dimensional continuous-time linear systems: stability and structure analysis. SIAM J. Control and Optim., 34:757-812, 1996. MR 97d:93077

[23] R.E.A.C. Paley and N. Wiener. Fourier Transforms in the Complex Domain. American Mathematical Society, Providence, Rhode Island, 1934. MR 98a:01023 (latest reprint)

[24] A. Pazy. Semi-Groups of Linear Operators and Applications to Partial Differential Equations. Springer-Verlag, Berlin, 1983. MR 85g:47061

[25] R. Rebarber. Conditions for the equivalence of internal and external stability for distributed parameter systems. IEEE Trans. Autom. Control, 38:994-998, 1993. MR 94b:93100

[26] R. Rebarber. Exponential stability of coupled beams with dissipative joints: a frequency domain approach. SIAM J. Control Optim., 33:1-28, 1995. MR 95i:93103

[27] R. Rebarber and S. Townley. Robustness and continuity of the spectrum for uncertain distributed parameter systems. Automatica, 31:1533-1546, 1995. MR 96f:93045

[28] D. Salamon. Infinite dimensional linear systems with unbounded control and observation: a functional analytic approach. Trans. Amer. Math. Soc., 300:383-431, 1987. MR 88d:93024

[29] D. Salamon. Realization theory in Hilbert space. Math. Systems Theory, 21:147-164, 1989. MR 89k:93038

[30] O.J. Staffans. Quadratic optimal control of stable well-posed linear systems. Trans. Amer. Math. Soc., 349:3679-3715, 1997. MR 97k:49011

[31] O.J. Staffans. Coprime factorizations and well-posed linear systems. SIAM J. Control Optim., 36:1268-1292, 1998. MR 99g:93049

[32] O.J. Staffans. Quadratic optimal control of well-posed linear systems. SIAM J. Control Optim., 37:131-164, 1998. MR 2000i:93046

[33] O.J. Staffans. Feedback representations of critical controls for well-posed linear systems. Internat. J. Robust and Nonlinear Control, 8:1189-1217, 1998. MR 99m:93043

[34] O.J. Staffans. On the distributed stable full information $H^{\infty}$ minimax problem. Internat. J. Robust and Nonlinear Control, 8:1255-1305, 1998. MR 99m:93034

[35] O.J. Staffans. Lax-Phillips scattering and well-posed linear systems. In Proceedings of the 7th IEEE Mediterranean Conference on Control and Systems, CD-ROM, Haifa, Israel, July 28-30, 1999.

[36] O.J. Staffans. Well-Posed Linear Systems. Book manuscript, 2002.

[37] E.G.F. Thomas: Vector valued integration with applications to the operator valued $H^{\infty}$ space, IMA J. on Math. Control and Inform., 14:109-136, 1997. MR 99d:28016

[38] G. Weiss. Admissibility of unbounded control operators. SIAM J. Control Optim., 27:527545, 1989. MR 90c:93060 
[39] G. Weiss. Admissible observation operators for linear semigroups. Israel J. Math., 65:17-43, 1989. MR 90g:47082

[40] G. Weiss. The representation of regular linear systems on Hilbert spaces. In Control and Estimation of Distributed Parameter Systems, vol. 91 of ISNM, eds. F. Kappel, K. Kunisch, W. Schappacher, pp. 401-416, Birkhäuser-Verlag, Basel, 1989. MR 91d:93026

[41] G. Weiss. Transfer functions of regular linear systems. Part I: Characterizations of regularity. Trans. Amer. Math. Soc., 342:827-854, 1994. MR 91f:93074

[42] G. Weiss. Regular linear systems with feedback. Math. Control, Signals and Systems, 7:23-57, 1994. MR 96i:93046

[43] G. Weiss. A powerful generalization of the Carleson measure theorem? In Open Problems in Math. Systems and Control Theory, eds. V. Blondel, E. Sontag, M. Vidyasagar, J. Willems, pp. 267-272, Springer-Verlag, London, 1999. MR 2000g:93003

[44] G. Weiss and R.F. Curtain. Dynamic stabilization of regular linear systems. IEEE Trans. Autom. Control, 42:4-21, 1997. MR 98d:93084

[45] M. Weiss and G. Weiss. Optimal control of stable weakly regular linear systems. Math. Control, Signals and Systems, 10:287-330, 1997. MR 99h:49037

[46] G. Weiss and M. Häfele. Repetitive control of MIMO systems using $H^{\infty}$ design. Automatica, 35:1185-1199, 1999. CMP 2001:12

[47] Y. Yamamoto. Realization theory of infinite-dimensional linear systems, parts I and II. Math. Systems Theory, 15:55-77,169-190, 1981. MR 83j:93031a; MR 83j:93031b

[48] K. Zhou, J. Doyle and K. Glover. Robust and Optimal Control. Prentice-Hall, Upper Saddle River, 1996.

Department of Mathematics, Åbo Akademi University, Fin-20500 Åbo, Finland

E-mail address: Olof.Staffans@abo.fi

$U R L:$ http://www.abo.fi/ ${ }^{\sim}$ staffans

Department of Electrical \& Electronic Engineering, imperial College of Science \& Technology, Exhibition Road, London SW7 2BT, United Kingdom

E-mail address: G.Weiss@ic.ac.uk 